\title{
Dual representation of lattice QCD with worldlines and worldsheets of Abelian color fluxes
}

\author{
Carlotta Marchis $^{*}$ and Christof Gattringer ${ }^{\dagger}$ \\ Graz Universität, Institut für Physik, Universitätsplatz, 5, 8010 Graz, Austria
}

(Received 21 December 2017; published 16 February 2018)

\begin{abstract}
We present a new dual representation for lattice QCD in terms of wordlines and worldsheets. The exact reformulation is carried out using the recently developed Abelian color flux method where the action is decomposed into commuting minimal terms that connect different colors on neighboring sites. Expanding the Boltzmann factors for these commuting terms allows one to reorganize the gauge field contributions according to links such that the gauge fields can be integrated out in closed form. The emerging constraints give the dual variables the structure of worldlines for the fermions and worldsheets for the gauge degrees of freedom. The partition sum has the form of a strong coupling expansion, and with the Abelian color flux approach discussed here all coefficients of the expansion are known in closed form. We present the dual form for three cases: pure SU(3) lattice gauge theory, strong coupling QCD and full QCD, and discuss in detail the constraints for the color fluxes and their physical interpretation.
\end{abstract}

DOI: 10.1103/PhysRevD.97.034508

\section{INTRODUCTION}

An important strategy in theoretical physics is to find different representations of a system, such that after rewriting a model in terms of new degrees of freedom different physical aspects are revealed or new methods can be applied. In the context of lattice field theories exact transformations to representations in terms of worldlines for matter fields and worldsheets for gauge degrees of freedom have been studied in recent years (see, e.g., the reviews at the annual lattice conferences [1-4]). A strong motivation for this line of work is the sign problem at finite chemical potential, which in some models can be overcome with worldline/worldsheet representations, such that finite density simulations become accessible. However, recently also more abstract questions were addressed concerning the form of the constraints for the dual variables (i.e., worldlines and worldsheets) for different symmetries of the conventional representation.

Finding dual representations for theories with Abelian symmetries is essentially a closed case, see, e.g., the standard review [5]. For many Abelian systems a second transformation to yet another set of variables allows one to solve all constraints and to arrive at a completely dual form

\footnotetext{
carla.marchis@uni-graz.at

christof.gattringer@uni-graz.at
}

Published by the American Physical Society under the terms of the Creative Commons Attribution 4.0 International license. Further distribution of this work must maintain attribution to the author(s) and the published article's title, journal citation, and DOI. Funded by SCOAP ${ }^{3}$. in the Kramers-Wannier sense [6]. However, for nonAbelian symmetries the situation is far less advanced. The key technique is strong coupling expansion which has been explored since the earliest days of lattice field theory [7-11]. Recently, diagrammatic representations in terms of worldlines and worldsheets for QCD and QCDlike lattice field theories have seen a prominent revival, see, e.g. [12-23], mostly driven by the quest for finding new representations to solve the aforementioned sign problem of QCD. However, so far no real and positive finite density representations were found (including the approach presented here), and obviously new concepts, such as partial resummations are needed for possible applications in finite density simulations.

In this paper we present a dual representation of lattice QCD in terms of worldlines and worldsheets based on the recently introduced "Abelian color flux $(A C F)$ approach" [21-23]. While most approaches to strong coupling representations of non-Abelian theories rely on group integrals, often in the form of character expansion, the ACF approach decomposes the action into its smallest possible units, which are terms that connect different color indices on neighboring sites of the lattice. These objects are either complex numbers for the gauge field action or Grassmann bilinears for the fermions and thus commute in both cases. After expanding the individual Boltzmann factors one can reorder all terms and organize them with respect to links, such that they can be integrated over with the link-based Haar measure. No long range interdependencies of the integrals emerge, and all terms of the ACF form of the strong coupling expansion are obtained as closed expressions. We stress at this point that some of the weights have a 
negative sign, so without some form of resummation our representation cannot be directly used in a Monte Carlo simulation.

In this paper we focus on working out the ACF formulation for QCD, starting with the simpler cases of pure $\mathrm{SU}(3)$ gauge theory and strong coupling QCD, and deriving from those two limiting cases the full dual form of lattice QCD in terms of worldlines and worldsheets. We discuss in detail the form of the constraints that emerge for our dual degrees of freedom and show that they have the form of a conservation law for fluxes of all three colors ("color conservation constraints") and a second set of constraints that ensure the equal distribution of flux among the colors ("color exchange constraints"). We discuss the implications and geometrical interpretation of the constraints for all three cases we consider, i.e., pure SU(3) gauge theory, strong coupling QCD and full QCD. For the case of strong coupling QCD we discuss the behavior of the strong coupling baryon loops and show that they are closely related to free staggered fermions for the baryons, embedded in a background of local fermion monomials with positive weights.

\section{SU(3) LATTICE GAUGE THEORY}

We start the presentation with deriving the worldsheet representation for pure SU(3) lattice gauge theory. We work with the Wilson gauge action

$$
S_{G}[U]=-\frac{\beta}{3} \sum_{x, \mu<\nu} \operatorname{Re} \operatorname{Tr} U_{x, \mu} U_{x+\hat{\mu}, \nu} U_{x+\hat{\nu}, \mu}^{\dagger} U_{x, \nu}^{\dagger},
$$

where $U_{x, \mu} \in \mathrm{SU}(3)$ are the dynamical degrees of freedom of the theory. They live on the links $(x, \mu)$ of a fourdimensional lattice with periodic boundary conditions. The size of the lattice, i.e., the number of sites will be denoted by $V$. The partition function $Z$ is obtained by integrating the Boltzmann factor $e^{-S_{G}[U]}$ with the product of SU(3) Haar measures $\int D[U]=\prod_{x, \mu} \int_{\mathrm{SU}(3)} d U_{x, \mu}$,

$$
Z=\int D[U] e^{-S_{G}[U]}
$$

As already outlined in the Introduction, the first step of our approach consists of writing explicitly the trace and the matrix multiplications in the action (1) as sums over color indices for products of gauge link elements $U_{x, \mu}^{a b}$,

$$
\begin{aligned}
S_{G}[U]= & -\frac{\beta}{6} \sum_{x, \mu<\nu} \sum_{a, b, c, d=1}^{3}\left[U_{x, \mu}^{a b} U_{x+\hat{\mu}, \nu}^{b c} U_{x+\hat{\nu}, \mu}^{d c \star} U_{x, \nu}^{a d} \star\right. \\
& \left.+U_{x, \mu}^{a b \star} U_{x+\hat{\mu}, \nu}^{b c} U_{x+\hat{\nu}, \mu}^{d c} U_{x, \nu}^{a d}\right] .
\end{aligned}
$$

The two products $U_{x, \mu}^{a b} U_{x+\hat{\mu}, \nu}^{b c} U_{x+\hat{\nu}, \mu}^{d c} U_{x, \nu}^{a d} \star \quad$ and $U_{x, \mu}^{a b \star} U_{x+\hat{\mu}, \nu}^{b c \star} U_{x+\hat{\nu}, \mu}^{d c} U_{x, \nu}^{a d}$ are the objects we refer to as the

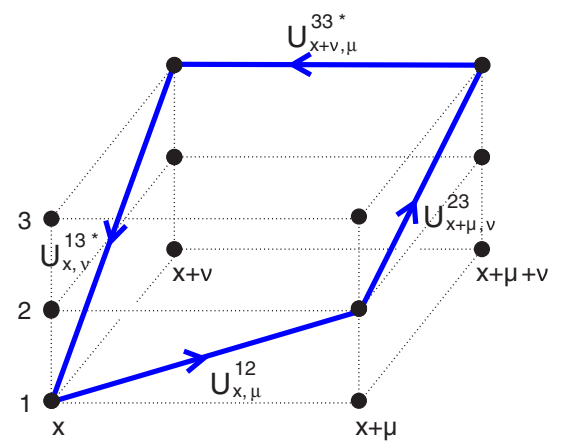

FIG. 1. Graphical representation of the $(1,2,3,3)-\mathrm{ACC}$, which explicitly is given by $U_{x, \mu}^{12} U_{x+\hat{\mu}, \nu}^{23} U_{x+\hat{\nu}, \mu}^{33} U_{x, \nu}^{13}$. This ACC closes around the plaquette $(x, \mu \nu)$ running through the sequence $(1,2,3,3)$ of color indices at the corners of the plaquette. In the graphical representation the color degrees of freedom are shown as three distinct layers of the space-time lattice, labeled with 1,2, and 3 on the lhs of the plot. Each of the matrix elements $U_{x, \mu}^{a b}$ constituting the ACC is represented by an arrow along the corresponding link $(x, \mu)$ connecting color $a$ with color $b$. For complex conjugate matrix elements the link is run through with negative orientation.

"Abelian color cycles" (ACCs) [21]. They are products of complex numbers and can be interpreted as paths in color space closing around plaquettes. In space-time we label the plaquettes $(x, \mu \nu)$ with the site $x$ in their lower left corner and the two directions $\mu<\nu$. The labeling of the ACCs is then completed by providing the values $(a, b, c, d)$ of the color indices at the four corners of the plaquette which determine the path in color space.

To give an example, in Fig. 1 we graphically illustrate the $(1,2,3,3)$-ACC which in explicit form is given by $U_{x, \mu}^{12} U_{x+\hat{\mu}, \nu}^{23} U_{x+\hat{\nu}, \mu}^{33 \star} U_{x, \nu}^{13}{ }^{\star}$. The color degrees of freedom are represented by using a lattice with three layers, which in the figure we sketch in light grey as three copies of the plaquette we consider. The terms in the $(1,2,3,3)$-ACC then all have a simple graphical representation: The factor $U_{x, \mu}^{12}$ is represented by an arrow connecting the color index 1 at $x$ with the color index 2 at $x+\hat{\mu}$. The factor $U_{x+\hat{\mu}, \nu}^{23}$ then continues from color 2 to color 3 along the link from $x+\hat{\mu}$ to $x+\hat{\mu}+\hat{\nu}$. Link matrix elements with a complex conjugation are interpreted as running in a negative direction, such that $U_{x+\hat{\nu}, \mu}^{33}$ leads from color 3 at $x+\hat{\mu}+\hat{\nu}$ to color 3 at $x+\hat{\nu}$ and $U_{x, \nu}^{13} \star$ closes the loop leading from color 3 at $x+\hat{\nu}$ to color 1 at $x$.

The rule of reverting the orientation with complex conjugation implies that the ACCs in the second summand of (3) run around the plaquette $(x, \mu \nu)$ with a mathematically negative orientation. Since for SU(3) there are three different possible choices for the color at every corner of the plaquette there is a total of $3^{4}=81$ different ACCs, each contributing with both orientations to (3).

The ACC decomposition (3) of the action (1) allows us to completely factorize the Boltzmann factor and to proceed 
with the dualization of the theory along the lines of the construction for Abelian gauge fields (see, e.g., [24,25]),

$$
\begin{aligned}
Z= & \int D[U] \prod_{x, \mu<\nu} \prod_{a, b, c, d} e^{\frac{\beta}{6}\left(U_{x, \mu}^{a b} U_{x+\hat{\mu}, \nu}^{b c} U_{x+\nu, \mu}^{d c \star} U_{x, \nu}^{a d \star}+\text { c.c. }\right)} \\
= & \int D[U] \prod_{x, \mu<\nu} \prod_{a, b, c, d} \sum_{n_{x, \mu \nu}^{a b c d}=0}^{\infty} \sum_{\bar{n}_{x, \mu \nu}^{a b c d}=0}^{\infty} \frac{(\beta / 6)^{n_{x, \mu \nu}^{a b c d}+\bar{n}_{x, \mu \nu}^{a b c d}}}{n_{x, \mu \nu}^{a b c d} ! \bar{n}_{x, \mu \nu}^{a b c d} !} \\
& \left.\times\left(U_{x, \mu}^{a b} U_{x+\hat{\mu}, \nu}^{b c} U_{x+\hat{\nu}, \mu}^{d c \star} U_{x, \nu}^{a d \star}\right)^{n_{x, \nu \nu}^{a b c d}} \text { (c.c. }\right)^{\bar{n}_{x, \mu \nu}^{a b c d}} .
\end{aligned}
$$

In the first step we have written all sums in the exponents as products over the individual Boltzmann weights $e^{\frac{\beta}{6} U_{x, \mu}^{a b} U_{x+\mu, \nu}^{b c} U_{x+\hat{\nu}, \mu}^{d c \star} U_{x, \nu}^{a d \star}}$ and $e^{\frac{\beta}{6} b_{x, \mu}^{a b} \star} U_{x+\hat{\mu}, \nu}^{b c c} U_{x+\hat{\nu}, \mu}^{d c} U_{x, \nu}^{a d}$ for the ACCs with positive and negative orientation. In the second step we expand each factor in a Taylor series, thus introducing two sets of expansion indices assigned to the plaquettes: $n_{x, \mu \nu}^{a b c d} \in \mathbb{N}_{0}$ and $\bar{n}_{x, \mu \nu}^{a b c d} \in \mathbb{N}_{0}$ where the color indices $a, b, c$ and $d$ each can have the values 1,2 or 3 . The variables $n_{x, \mu \nu}^{a b c d}$ correspond to the units of flux with color indices $a, b, c, d$ around the plaquette $(x, \mu \nu)$ in a positive orientation, and $\bar{n}_{x, \mu \nu}^{a b c d}$ is used for flux with negative orientation.

All the factors in the sums in (4) are products of complex numbers, such that we can freely commute them and reorganize them as in the Abelian case. Ordering the terms with respect to the links $(x, \mu)$ where we will subsequently integrate them with the Haar measure, the partition sum assumes the form

$$
\begin{aligned}
Z= & \sum_{\{n, \bar{n}\}}\left[\prod_{x, \mu<\nu} \prod_{a, b, c, d} \frac{(\beta / 6)^{n_{x, \mu \nu}^{a b c d}+\bar{n}_{x, \mu \nu}^{a b c d}}}{n_{x, \mu \nu}^{a b c d} ! \bar{n}_{x, \mu \nu}^{a b c d} !}\right] \\
& \times\left[\prod_{x, \mu} \int d U_{x, \mu} \prod_{a, b}\left(U_{x, \mu}^{a b}\right)^{N_{x, \mu}^{a b}}\left(U_{x, \mu}^{a b}\right)^{\bar{N}_{x, \mu}^{a b}}\right],
\end{aligned}
$$

where for the sum over configurations of the variables $n_{x, \mu \nu}^{a b c d}, \bar{n}_{x, \mu \nu}^{a b c d} \in \mathbb{N}_{0}$ we introduced the short hand notation $\sum_{\{n, \bar{n}\}}=\prod_{x, \mu<\nu} \prod_{a, b, c, d=1}^{3} \sum_{n_{x, \mu \nu}^{a b c d}=0}^{\infty} \sum_{\bar{n}_{x, \mu \nu}^{a b c d}=0}^{\infty}$. The integer valued powers $N_{x, \mu \nu}^{a b c d}$ and $\bar{N}_{x, \mu \nu}^{a b c d}$ collect all $n_{x, \mu \nu}^{a b c d}$ and $\bar{n}_{x, \mu \nu}^{a b c d}$ where the matrix elements $U_{x, \mu}^{a b}$ and $U_{x, \mu}^{a b \star}$ appear. Explicitly they are given by

$$
\begin{aligned}
& N_{x, \mu}^{a b}=\sum_{\nu: \mu<\nu} n_{x, \mu \nu}^{a b s s}+\bar{n}_{x-\hat{\nu}, \mu \nu}^{s s b a}+\sum_{\rho: \mu>\rho} \bar{n}_{x, \rho \mu}^{a s s b}+n_{x-\hat{\rho}, \rho \mu}^{s a b s}, \\
& \bar{N}_{x, \mu}^{a b}=\sum_{\nu: \mu<\nu} \bar{n}_{x, \mu \nu}^{a b s s}+n_{x-\hat{\nu}, \mu \nu}^{s s b a}+\sum_{\rho: \mu>\rho} n_{x, \rho \mu}^{a s s b}+\bar{n}_{x-\hat{\rho}, \rho \mu}^{s a b s} .
\end{aligned}
$$

The label $s$ introduced here is the short hand notation for an independent summation over all color indices replaced by $s$, e.g., $n_{x, \rho \mu}^{a s s b} \equiv \sum_{c, d} n_{x, \rho \mu}^{a c d b}$.

The Haar measure integration in (5) is now done using an explicit parametrization for the SU(3) matrices [26],

$$
U_{x, \mu}=\left(\begin{array}{ccc}
c_{1} c_{2} e^{i \phi_{1}} & s_{1} e^{i \phi_{3}} & c_{1} s_{2} e^{i \phi_{4}} \\
s_{2} s_{3} e^{-i \phi_{4}-i \phi_{5}}-s_{1} c_{2} c_{3} e^{i \phi_{1}+i \phi_{2}-i \phi_{3}} & c_{1} c_{3} e^{i \phi_{2}} & -c_{2} s_{3} e^{-i \phi_{1}-i \phi_{5}}-s_{1} s_{2} c_{3} e^{i \phi_{2}-i \phi_{3}+i \phi_{4}} \\
-s_{2} c_{3} e^{-i \phi_{2}-i \phi_{4}}-s_{1} c_{2} s_{3} e^{i \phi_{1}-i \phi_{3}+i \phi_{5}} & c_{1} s_{3} e^{i \phi_{5}} & c_{2} c_{3} e^{-i \phi_{1}-i \phi_{2}}-s_{1} s_{2} s_{3} e^{-i \phi_{3}+i \phi_{4}+i \phi_{5}}
\end{array}\right)
$$

The parametrization uses three angles $\theta_{x, \mu}^{(j)} \in[0, \pi / 2]$, $j=1,2,3$ and five phases $\phi_{x, \mu}^{(j)} \in[-\pi, \pi], j=1 \ldots 5$. In (8) we use the abbreviations $c_{j}=\cos \theta_{x, \mu}^{(j)}, s_{j}=\sin \theta_{x, \mu}^{(j)}$ and $\phi_{j}=\phi_{x, \mu}^{(j)}$. For the parametrization (8) the normalized Haar measure is given by

$$
d U_{x, \mu}=16 d \theta_{1} c_{1}^{3} s_{1} d \theta_{2} c_{2} s_{2} d \theta_{3} c_{3} s_{3} \prod_{j=1}^{5} \frac{d \phi_{j}}{2 \pi} .
$$

We will see below that the integration over the angles $\phi_{x, \mu}^{(j)}, j=1,2 \ldots 5$ will give rise to constraints for the variables $n_{x, \mu \nu}^{a b c d}$ and $\bar{n}_{x, \mu \nu}^{a b c d}$. In order to give these constraints a transparent form it is useful to perform the change of variables

$$
\begin{aligned}
n_{x, \mu \nu}^{a b c d}-\bar{n}_{x, \mu \nu}^{a b c d}=p_{x, \mu \nu}^{a b c d}, \quad & p_{x, \mu \nu}^{a b c d} \in \mathbb{Z}, \\
n_{x, \mu \nu}^{a b c d}+\bar{n}_{x, \mu \nu}^{a b c d}=\left|p_{x, \mu \nu}^{a b c d}\right|+2 l_{x, \mu \nu}^{a b c d}, \quad & l_{x, \mu \nu}^{a b c d} \in \mathbb{N}_{0},
\end{aligned}
$$

and instead of summing over the configurations of the $n_{x, \mu \nu}^{a b c d}$ and $\bar{n}_{x, \mu \nu}^{a b c d}$ to sum over configurations of the $p_{x, \mu \nu}^{a b c d}$ and $l_{x, \mu \nu}^{a b c d}$. The sets of variables $p_{x, \mu \nu}^{a b c d} \in \mathbb{Z}$ and $l_{x, \mu \nu}^{a b c d} \in \mathbb{N}_{0}$, which are both assigned to the plaquettes of the lattice will be the new dynamical dual degrees of freedom that we use in the partition sum after integrating out the conventional fields $U_{x, \mu}$.

The $p_{x, \mu \nu}^{a b c d}$ will be subject to constraints, and for understanding these constraints it is important to discuss the geometrical interpretation of the $p_{x, \mu \nu}^{a b c d}$ : From the definition (10) and the interpretation of the $n_{x, \mu \nu}^{a b c d}\left(\bar{n}_{x, \mu \nu}^{a b c d}\right)$ as the activation numbers for $(a, b, c, d)$-ACCs with a positive (negative) orientation it is clear that the new variables $p_{x, \mu \nu}^{a b c d}$ activate $\left|p_{x, \mu \nu}^{a b c d}\right|$ units of flux for the $(a, b, c, d)$-ACC on the plaquette $(x, \mu \nu)$, with the orientation of the flux given by the sign of the $p_{x, \mu \nu}^{a b c d}$. We refer to the $p_{x, \mu \nu}^{a b c d}$ as "cycle occupation numbers". The $l_{x, \mu \nu}^{a b c d}$ are not subject to constraints, and we simply refer to them as "auxiliary plaquette variables". 
For further simplification it is convenient to introduce the link fluxes

$J_{x, \mu}^{a b}=\sum_{\nu: \mu<\nu}\left[p_{x, \mu \nu}^{a b s s}-p_{x-\hat{\nu}, \mu \nu}^{s s b a}\right]-\sum_{\rho: \mu>\rho}\left[p_{x, \rho \mu}^{a s s b}-p_{x-\hat{\rho}, \rho \mu}^{s a b s}\right]$,

and the auxiliary link sums

$$
\begin{aligned}
S_{x, \mu}^{a b}= & \sum_{\nu: \mu<\nu}\left[\left|p_{x, \mu \nu}^{a b s s}\right|+\left|p_{x-\hat{\nu}, \mu \nu}^{s s b a}\right|+2\left(l_{x, \mu \nu}^{a b s s}+l_{x-\hat{\nu}, \mu \nu}^{s s b a}\right)\right] \\
& +\sum_{\rho: \mu>\rho}\left[\left|p_{x, \rho \mu}^{a s s b}\right|+\left|p_{x-\hat{\rho}, \rho \mu}^{s a b s}\right|+2\left(l_{x, \rho \mu}^{a s s b}+l_{x-\hat{\rho}, \rho \mu}^{s a b s}\right)\right] .
\end{aligned}
$$

We will see that only the fluxes $J_{x, \mu}^{a b}$ will appear in the constraints, and we thus have to extend our geometrical interpretation of the dual variables to these objects: $J_{x, \mu}^{a b}$ is the total flux from color $a$ on site $x$ to color $b$ on site $x+\hat{\mu}$. This flux receives contributions from all the ACCs that are attached to the link $(x, \mu)$ and that contain the path from color $a$ to $b$ along that link. So, if we consider the plaquette $(x, \mu \nu)$, with $\mu<\nu$, we have nine different ACCs that contribute to that flux, namely the ones corresponding to the cycle occupation numbers $p_{x, \mu \nu}^{a b e f}$, where $a$ and $b$ are the color indices which we fix at $x$ and $x+\hat{\mu}$. The colors $e$ and $f$ determine the ACC at the remaining two corners of $(x, \mu \nu)$. Both, $e$ and $f$ can be chosen independently from the set $\{1,2,3\}$ such that we have $3^{2}=9$ possibilities. Since the flux of the ACCs on the plaquette $(x, \mu \nu)$ has a positive orientation along the link $(x, \mu)$, these nine ACCs contribute with a positive sign in the definition (12) of the fluxes $J_{x, \mu}^{a b}$. However, $J_{x, \mu}^{a b}$ receives contributions from all plaquettes that contain the link $(x, \mu)$, such as the plaquettes $(x, \rho \mu)$ with $\rho<\mu$. For this case $J_{x, \mu}^{a b}$ receives contributions from the nine ACCs with occupation numbers $p_{x, \rho \mu}^{a e f b}$, but here the link $(x, \mu)$ is run through with a negative orientation, such that the $p_{x, \rho \mu}^{a e f b}$ contribute with a negative sign. For the remaining plaquettes that contain the link $(x, \mu)$ and thus contribute to $J_{x, \mu}^{a b}$ an analogous discussion holds.

In order to illustrate the geometrical interpretation of the cycle occupation numbers that contribute to a given $J_{x, \mu}^{a b}$, in Fig. 2 we illustrate the contributions from a plaquette $(x, \rho \mu)$ with $\rho<\mu$ to $J_{x, \mu}^{12}$. The $(1,2)$ flux on the link $(x, \mu)$ is fixed and represented with a full arrow pointing in the

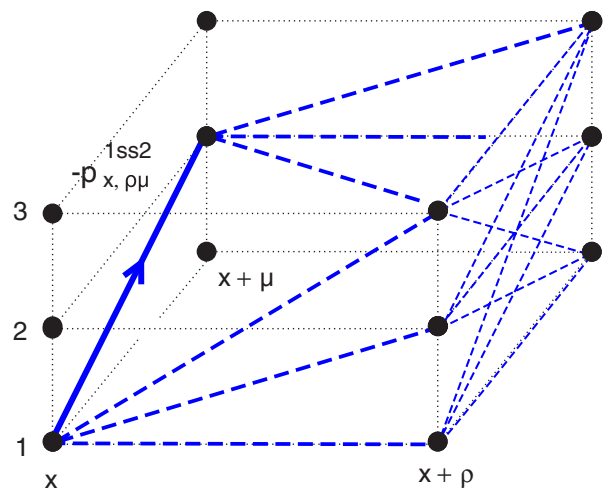

FIG. 2. Graphical illustration of the sum $-p_{x, \rho \mu}^{1 s s 2}$ contributing to the flux $J_{x, \mu}^{12}$.

positive $\mu$ direction. The nine ACCs on the plaquette $(x, \rho \mu)$ that contribute to this flux are represented with dashed lines in the figure. Since the ACCs on the plaquette $(x, \rho \mu)$ have a negative orientation of the flux on the link $(x, \mu)$, they contribute with a negative sign to the flux $J_{x, \mu}^{12}$.

Having introduced the fluxes $J_{x, \mu}^{a b}$ and the auxiliary sums $S_{x, \mu}^{a b}$ we can rewrite the integers $N_{x, \mu}^{a b}$ and $\bar{N}_{x, \mu}^{a b}$ that denote the powers of $U_{x, \mu}^{a b}$ and $U_{x, \mu}^{a b \star}$ in (5) in terms of the $J_{x, \mu}^{a b}$ and $S_{x, \mu}^{a b}$ as

$$
N_{x, \mu}^{a b}=\frac{S_{x, \mu}^{a b}+J_{x, \mu}^{a b}}{2}, \quad \bar{N}_{x, \mu}^{a b}=\frac{S_{x, \mu}^{a b}-J_{x, \mu}^{a b}}{2}
$$

Since in (5) the matrix elements $U_{x, \mu}^{a b}$ appear in the combination $\left(U_{x, \mu}^{a b}\right)^{N_{x, \mu}^{a b}}\left(U_{x, \mu}^{a b \star}\right)^{\bar{N}_{x, \mu}^{a b}}$ the form (14) separates the moduli and the phases of the matrix elements in a natural way.

However, an additional step is still required before we arrive at the final form of the terms in (5) where we can perform the Haar measure integration at each link. The problem is that some of the elements $U_{x, \mu}^{a b}$ of the matrix (8) are not in the simple form $U_{x, \mu}^{a b}=r_{x, \mu}^{a b} e^{i \varphi_{x, \mu}^{a b}}$, but are sums $U_{x, \mu}^{a b}=\rho_{x, \mu}^{a b} e^{i \alpha_{x, \mu}^{a b}}+\omega_{x, \mu}^{a b} e^{i \beta_{x, \mu}^{a b}}$. More specifically, in the parametrization (8) the $(a, b)=(2,1),(2,3),(3,1)$ and $(3,3)$ matrix elements are sums of two terms. For these entries we use the binomial theorem $(x+y)^{n}=\sum_{k=0}^{n}\left(\begin{array}{l}n \\ k\end{array}\right) x^{n-k} y^{k}$ and rewrite their contribution in the integrand of (5) as

$$
\begin{aligned}
& \left(U_{x, \mu}^{a b}\right)^{N_{x, \mu}^{a b}}\left(U_{x, \mu}^{a b}\right)^{\bar{N}_{x, \mu}^{a b}}=\left(\rho_{x, \mu}^{a b} e^{i \alpha_{x, \mu}^{a b}}+\omega_{x, \mu}^{a b} e^{i \beta_{x, \mu}^{a b}}\right)^{N_{x, \mu}^{a b}}\left(\rho_{x, \mu}^{a b} e^{-i \alpha_{x, \mu}^{a b}}+\omega_{x, \mu}^{a b} e^{-i \beta_{x, \mu}^{a b}}\right)^{\bar{N}_{x, \mu}^{a b}} \\
& =\sum_{m_{x, \mu}^{a b}=0}^{N_{x, \mu}^{a b}} \sum_{\bar{m}_{x, \mu}^{a b}=0}^{\bar{N}_{x, \mu}^{a b}}\left(\begin{array}{c}
N_{x, \mu}^{a b} \\
m_{x, \mu}^{a b}
\end{array}\right)\left(\begin{array}{c}
\bar{N}_{x, \mu}^{a b} \\
\bar{m}_{x, \mu}^{a b}
\end{array}\right)\left(\rho_{x, \mu}^{a b}\right)^{s_{x, \mu}^{a b}}\left(\omega_{x, \mu}^{a b}\right)^{S_{x, \mu}^{a b}-s_{x, \mu}^{a b}} e^{i \alpha_{x, \mu}^{a b} j_{x, \mu}^{a b}} e^{i \beta_{x, \mu}^{a b}\left(J_{x, \mu}^{a b}-j_{x, \mu}^{a b}\right)} \\
& \text { with } \quad m_{x, \mu}^{a b}=0,1 \ldots N_{x, \mu}^{a b}, \quad \bar{m}_{x, \mu}^{a b}=0,1 \ldots \bar{N}_{x, \mu}^{a b}, \\
& \text { and } \quad j_{x, \mu}^{a b} \equiv m_{x, \mu}^{a b}-\bar{m}_{x, \mu}^{a b}, \quad s_{x, \mu}^{a b} \equiv m_{x, \mu}^{a b}+\bar{m}_{x, \mu}^{a b} \text {. }
\end{aligned}
$$


Note that the new auxiliary variables $m_{x, \mu}^{a b}$ and $\bar{m}_{x, \mu}^{a b}$ which we use for the binomial decomposition of the matrix elements with $(a, b)=(2,1),(2,3),(3,1)$ and $(3,3)$ live on the links of the lattice.
To obtain the final result for the partition function we substitute the parametrization (8) and the Haar measure (9) in (5) and use the binomial decomposition (15). For the partition function we then obtain

$$
\begin{aligned}
& Z=2^{4 V} \sum_{\{p, l\}} \sum_{\{m, \bar{m}\}}\left[\prod_{x, \mu<\nu} \prod_{a, b, c, d} \frac{(\beta / 2)^{\left|p_{x, \mu \nu}^{a b c d}\right|+2 l_{x, \mu \nu}^{a b c d}}}{\left(\left|p_{x, \mu \nu}^{a b c d}\right|+l_{x, \mu \nu}^{a b c d}\right) ! l_{x, \mu \nu}^{a b c d} !}\right]\left[\prod_{x, \mu}(-1)^{S_{x, \mu}^{23}+S_{x, \mu}^{31}+s_{x, \mu}^{21}+S_{x, \mu}^{33}}\right]\left[\prod_{x, \mu} \prod_{a=2,3} \prod_{b=1,3}\left(\begin{array}{c}
N_{x, \mu}^{a b} \\
m_{x, \mu}^{a b}
\end{array}\right)\left(\begin{array}{c}
\bar{N}_{x, \mu}^{a b} \\
\bar{m}_{x, \mu}^{a b}
\end{array}\right)\right]
\end{aligned}
$$

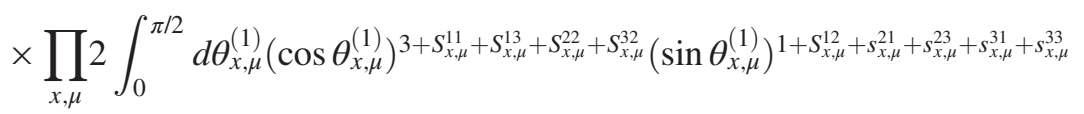

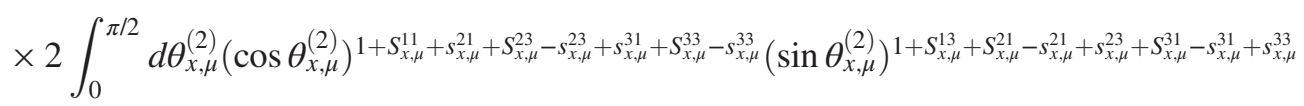

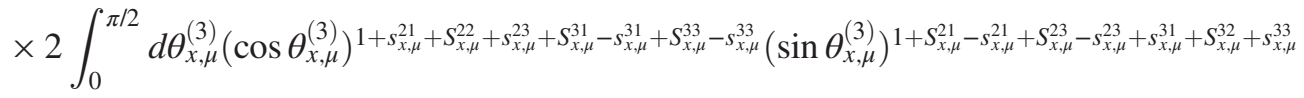

$$
\begin{aligned}
& \times \int_{0}^{2 \pi} \frac{d \phi_{x, \mu}^{(1)}}{2 \pi} e^{i \phi_{x, \mu}^{(1)}\left[J_{x, \mu}^{11}-J_{x, \mu}^{23}-J_{x, \mu}^{33}+j_{x, \mu}^{21}+j_{x, \mu}^{23}+j_{x, \mu}^{31}+j_{x, \mu}^{33}\right]} \int_{0}^{2 \pi} \frac{d \phi_{x, \mu}^{(2)}}{2 \pi} e^{i \phi_{x, \mu}^{(2)}\left[x_{x, \mu}^{22,}-J_{x, \mu}^{31}-J_{x, \mu}^{33}+j_{x, \mu}^{21}+j_{x, \mu}^{23}+j_{x, \mu}^{31}+j_{x, \mu}^{33}\right]} \\
& \times \int_{0}^{2 \pi} \frac{d \phi_{x, \mu}^{(3)}}{2 \pi} e^{i \phi_{x, \mu}^{(3)}\left[x_{x, \mu}^{12}-j_{x, \mu}^{21}-j_{x, \mu}^{23}-j_{x, \mu}^{31}-j_{x, \mu}^{33}\right]} \int_{0}^{2 \pi} \frac{d \phi_{x, \mu}^{(4)}}{2 \pi} e^{i \phi_{x, \mu}^{(4)}\left[J_{x, \mu}^{13}-J_{x, \mu}^{21}-J_{x, \mu}^{31}+j_{x, \mu}^{21}+j_{x, \mu}^{23}+j_{x, \mu}^{31}+j_{x, \mu}^{33}\right]} \\
& \times \int_{0}^{2 \pi} \frac{d \phi_{x, \mu}^{(5)}}{2 \pi} e^{i \phi_{x, \mu}^{(5)}\left[\left[_{x, \mu}^{32}-J_{x, \mu}^{21}-J_{x, \mu}^{23}+j_{x, \mu}^{21}+j_{x, \mu}^{23}+j_{x, \mu}^{31}+j_{x, \mu}^{33}\right]\right.}
\end{aligned}
$$

where we introduced the short hand notation

$$
\sum_{\{p\}}=\prod_{x, \mu<\nu} \prod_{a, b, c, d} \sum_{p_{x, \mu \nu}^{a b c d}=-\infty}^{\infty}, \quad \sum_{\{l\}}=\prod_{x, \mu<\nu} \prod_{a, b, c, d} \sum_{l_{x, y \nu}^{a b d}=0}^{\infty},
$$

for the sums over configurations of the cycle occupation numbers $p_{x, \mu \nu}^{a b c d} \in \mathbb{Z}$ and the auxiliary plaquette variables $l_{x, \mu \nu}^{a b c d} \in \mathbb{N}_{0}$, as well as

$$
\sum_{\{m, \bar{m}\}}=\prod_{x, \mu} \prod_{a=2,3} \prod_{b=1,3} \sum_{m_{x, \mu}^{a b}=0}^{N_{x, \mu}^{a b}} \sum_{\bar{m}_{x, \mu}^{a b}=0}^{\bar{N}_{x, \mu}^{a b}},
$$

for the sums over configurations of the link based auxiliary variables $m_{x, \mu}^{a b}$ and $\bar{m}_{x, \mu}^{a b}$ used in the binomial decomposition (15).

A key step of our approach is that now, after expanding the Boltzmann factors for the individual ACCs and reorganizing all contributions with respect to links, in (16) we can solve all Haar measure integrals in closed form. The integrals over the angles $\theta_{x, \mu}^{(j)}$ give rise to beta functions,

$2 \int_{0}^{\pi / 2} d \theta(\cos \theta)^{n+1}(\sin \theta)^{m+1}=B\left(\frac{n}{2}+1 \mid \frac{m}{2}+1\right)$.

The integrals over the phase factors $\phi_{x, \mu}^{(j)}$ in (16) give rise to Kronecker deltas [we use the notation $\delta(n) \equiv \delta_{n, 0}$ ] which impose constraints on the dual variables.
Putting together all terms we can write the dual form of the partition function of pure $\mathrm{SU}(3)$ lattice gauge theory in the form

$$
Z=\sum_{\{p\}} W_{G}[p] C_{G}[p]
$$

where we have defined the link-based gauge constraints $C_{G}[p]$ that are given by

$$
\begin{aligned}
C_{G}[p]= & \prod_{x, \mu} \delta\left(J_{x, \mu}^{12}+J_{x, \mu}^{13}-J_{x, \mu}^{21}-J_{x, \mu}^{31}\right) \\
& \times \delta\left(J_{x, \mu}^{21}+J_{x, \mu}^{23}-J_{x, \mu}^{12}-J_{x, \mu}^{32}\right) \\
& \times \delta\left(J_{x, \mu}^{11}+J_{x, \mu}^{12}-J_{x, \mu}^{23}-J_{x, \mu}^{33}\right) \\
& \times \delta\left(J_{x, \mu}^{31}+J_{x, \mu}^{33}-J_{x, \mu}^{12}-J_{x, \mu}^{22}\right)
\end{aligned}
$$

At every link $(x, \mu)$ we have four individual constraints that come from integrating the four phases $\phi_{x, \mu}^{(j)}, j=1,2,4,5$ of the representation (8) giving rise to the four Kronecker deltas shown in (21). Here we have already taken into account another constraint generated by the $\phi_{x, \mu}^{(3)}$ integral in (16) which implements the relation

$$
j_{x, \mu}^{21}+j_{x, \mu}^{23}+j_{x, \mu}^{31}+j_{x, \mu}^{33}=J_{x, \mu}^{12},
$$

that connects $J_{x, \mu}^{12}$ to the auxiliary currents $j_{x, \mu}^{a b}=$ $m_{x, \mu}^{a b}-\bar{m}_{x, \mu}^{a b}$ for the variables $m_{x, \mu}^{a b}, \bar{m}_{x, \mu}^{a b}$ introduced in 
(15) for the binomial decomposition for the $(2,1)$, $(2,3),(3,1)$ and $(3,3)$ matrix elements. To obtain (21) we have used (22) to replace the combination $j_{x, \mu}^{21}+j_{x, \mu}^{23}+j_{x, \mu}^{31}+j_{x, \mu}^{33}$ by $J_{x, \mu}^{12}$ in the integrals over $\phi_{x, \mu}^{(j)}, j=1,2,4,5$ in (16). In the final form (21) of $C_{G}[p]$ we show only the corresponding four constraints, while the constraint (22) is included in the weight factor $W_{G}[p]$.

The weight factor $W_{G}[p]$ in (20) is itself a sum $\sum_{\{l, m, \bar{m}\}}$ over configurations of the auxiliary plaquette variables $l_{x, \mu \nu}^{a b c d}$ and the link-based auxiliary variables $m_{x, \mu}^{a b}$ and $\bar{m}_{x, \mu}^{a b}$ used for the binomial decomposition in (15),

$$
\begin{aligned}
& W_{G}[p]=2^{4 V} \sum_{\{l, m, \bar{m}\}}\left[\prod_{x, \mu} \delta\left(J_{x, \mu}^{12}-j_{x, \mu}^{21}-j_{x, \mu}^{23}-j_{x, \mu}^{31}-j_{x, \mu}^{33}\right)\right]\left[\prod_{x, \mu}(-1)^{J_{x, \mu}^{12}+j_{x, \mu}^{23}+J_{x, \mu}^{31}-j_{x, \mu}^{23}-j_{x, \mu}^{31}}\right] \\
& \times\left[\prod_{x, \mu} \prod_{a=2,3} \prod_{b=1,3}\left(\begin{array}{c}
N_{x, \mu}^{a b} \\
m_{x, \mu}^{a b}
\end{array}\right)\left(\begin{array}{c}
\bar{N}_{x, \mu}^{a b} \\
\bar{m}_{x, \mu}^{a b}
\end{array}\right)\right]\left[\prod_{x, \mu<\nu} \prod_{a, b, c, d} \frac{(\beta / 2)^{\left|p_{x, \mu \nu}^{a b c d}\right|+2 l_{x, \mu \nu}^{a b c d}}}{\left(\left|p_{x, \mu \nu}^{a b c d}\right|+l_{x, \mu \nu}^{a b c d}\right) ! l_{x, \mu \nu}^{a b c d !} !}\right] \\
& \times\left[\prod_{x, \mu} B\left(\frac{S_{x, \mu}^{11}+S_{x, \mu}^{13}+S_{x, \mu}^{22}+S_{x, \mu}^{32}}{2}+2 \mid \frac{S_{x, \mu}^{12}+s_{x, \mu}^{21}+s_{x, \mu}^{23}+s_{x, \mu}^{31}+s_{x, \mu}^{33}}{2}+1\right)\right. \\
& \times B\left(\frac{S_{x, \mu}^{11}+s_{x, \mu}^{21}+S_{x, \mu}^{23}-s_{x, \mu}^{23}+s_{x, \mu}^{31}+S_{x, \mu}^{33}-s_{x, \mu}^{33}}{2}+1 \mid \frac{S_{x, \mu}^{13}+S_{x, \mu}^{21}-s_{x, \mu}^{21}+s_{x, \mu}^{23}+S_{x, \mu}^{31}-s_{x, \mu}^{31}+s_{x, \mu}^{33}}{2}+1\right) \\
& \left.\times B\left(\frac{s_{x, \mu}^{21}+S_{x, \mu}^{22}+s_{x, \mu}^{23}+S_{x, \mu}^{31}-s_{x, \mu}^{31}+S_{x, \mu}^{33}-s_{x, \mu}^{33}}{2}+1 \mid \frac{S_{x, \mu}^{21}-s_{x, \mu}^{21}+S_{x, \mu}^{23}-s_{x, \mu}^{23}+s_{x, \mu}^{31}+S_{x, \mu}^{32}+s_{x, \mu}^{33}}{2}+1\right)\right] .
\end{aligned}
$$

The configurations of the $m_{x, \mu}^{a b}$ and $\bar{m}_{x, \mu}^{a b}$ are restricted by the Kronecker delta constraints, which implement (22) at every link $(x, \mu)$. In $W_{G}[p]$ we collect all weights from the expansion of the individual Boltzmann factors and the beta functions resulting from the Haar measure integrals. These weight factors are organized with respect to powers of the inverse gauge coupling $\beta$, i.e., the dual formulation in terms of ACC cycle occupation numbers which we develop here is a strong coupling expansion. A major advantage of the strong coupling series in terms of ACCs is that all weight factors at arbitrary orders of $\beta$ are known in a closed form: They are given in terms of factorials, binomial coefficients and beta functions (which can also be rewritten as fractions of factorials). We stress that in the form (23) there is an explicit sign factor. This factor comes from the minus signs in the parametrization (8) used for the $\mathrm{SU}(3)$ group elements. This implies that for a Monte Carlo simulation of the ACC dual form a strategy for partial resummation needs to be found.

Let us now come to the announced discussion of the constraints in (21). Understanding how the SU(3) symmetry of the conventional representation becomes manifest in terms of constraints for the dual variables is one of the key points of this paper. In (21) at each link $(x, \mu)$ the fluxes $J_{x, \mu}^{a b}$ are related to each other by four constraints implemented by Kronecker deltas. These relations read

$$
\begin{aligned}
& J_{x, \mu}^{12}+J_{x, \mu}^{13}=J_{x, \mu}^{21}+J_{x, \mu}^{31}, \\
& J_{x, \mu}^{21}+J_{x, \mu}^{23}=J_{x, \mu}^{12}+J_{x, \mu}^{32},
\end{aligned}
$$

$$
\begin{aligned}
& J_{x, \mu}^{11}+J_{x, \mu}^{12}=J_{x, \mu}^{23}+J_{x, \mu}^{33}, \\
& J_{x, \mu}^{31}+J_{x, \mu}^{33}=J_{x, \mu}^{12}+J_{x, \mu}^{22} .
\end{aligned}
$$

The relations (24)-(27) describe how the SU(3) gauge invariance of the conventional representation is encoded in constraints for the fluxes $J_{x, \mu}^{a b}$. These relations can be combined and reorganized in a way that makes the flow between the different color indices $a, b$ more transparent. On both sides of (24) we may add $J_{x, \mu}^{11}$ and on both sides of (25) we add $J_{x, \mu}^{22}$. Furthermore we can subtract (25) from (24) and add $J_{x, \mu}^{33}$ on both sides. This gives the following three relations:

$$
\begin{aligned}
& J_{x, \mu}^{11}+J_{x, \mu}^{12}+J_{x, \mu}^{13}=J_{x, \mu}^{11}+J_{x, \mu}^{21}+J_{x, \mu}^{31}, \\
& J_{x, \mu}^{21}+J_{x, \mu}^{22}+J_{x, \mu}^{23}=J_{x, \mu}^{12}+J_{x, \mu}^{22}+J_{x, \mu}^{32}, \\
& J_{x, \mu}^{31}+J_{x, \mu}^{32}+J_{x, \mu}^{33}=J_{x, \mu}^{13}+J_{x, \mu}^{23}+J_{x, \mu}^{33} .
\end{aligned}
$$

The first relation (28) implies that for all links $(x, \mu)$ the flux out of color 1 at $x$ equals the flux into color 1 at $x+\hat{\mu}$. The other two relations imply the same conservation law for color 2 and color 3 . Thus for all three colors $a=1,2,3$ we have the constraint that along each link the flux out of color $a$ has to match the flux into that color $a$. Consequently the constraints (28)-(30) imply that for each color $a$ the flux that runs through $a$ is the same at all sites. Thus we refer to (28)-(30) as the "color conservation constraints". 

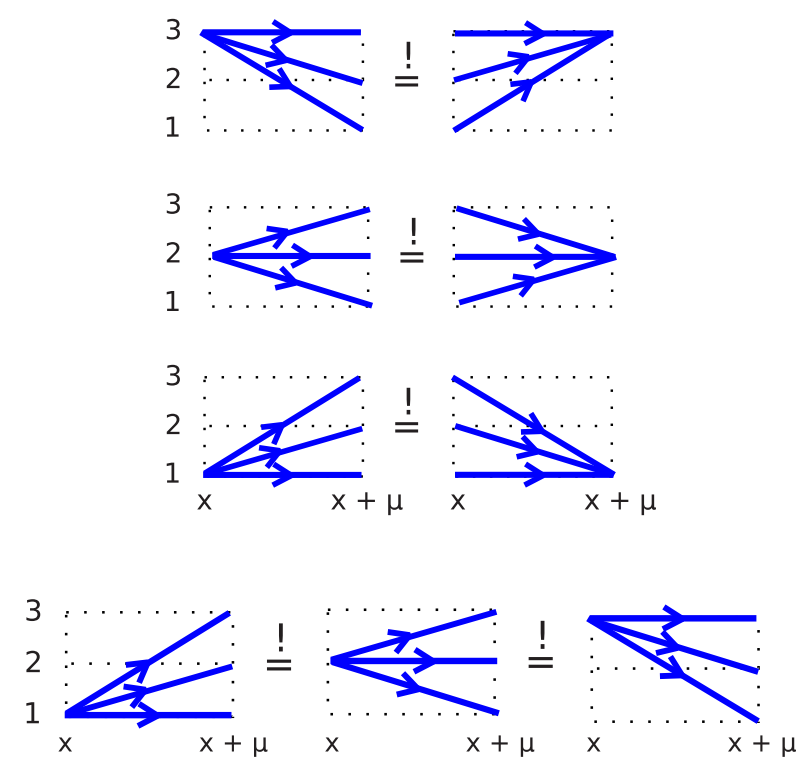

FIG. 3. Schematic representation of the three color conservation constraints (28)-(30) (top three plots) and the color exchange constraint (31) (bottom plot). They impose relations between the fluxes $J_{x, \mu}^{a b}$ and admissible configurations of the cycle occupation numbers $p_{x, \mu \nu}^{a b c d}$ have to respect these constraints.

These three constraints are illustrated in the first three plots of Fig. 3.

A second type of constraints among the $J_{x, \mu}^{a b}$ is obtained by adding $J_{x, \mu}^{13}$ on both sides of (26) and adding $J_{x, \mu}^{32}$ on both sides of (27). The right-hand sides of the resulting two equations are then replaced using (29) and (30), and we can summarize the resulting relations as

$$
\begin{aligned}
J_{x, \mu}^{11}+J_{x, \mu}^{12}+J_{x, \mu}^{13} & =J_{x, \mu}^{21}+J_{x, \mu}^{22}+J_{x, \mu}^{23} \\
& =J_{x, \mu}^{31}+J_{x, \mu}^{32}+J_{x, \mu}^{33} .
\end{aligned}
$$

This constraint implies that the flux that flows out of a color $a$ along a link $(x, \mu)$ is the same for all three colors $a$. Thus if flux is exchanged between the colors along some link, the exchanged flux has to be the same for all three colors. We refer to (31) as "color exchange constraints".

The relations (28)-(31) enforce constraints among the fluxes $J_{x, \mu}^{a b}$ that have to be obeyed at every link $(x, \mu)$. The fluxes $J_{x, \mu}^{a b}$ defined in (12) depend only on the cycle occupation numbers $p_{x, \mu \nu}^{a b c d} \in \mathbb{Z}$, and thus only this set of dual variables is subject to the constraints-the auxiliary plaquette variables $l_{x, \mu \nu}^{a b c d} \in \mathbb{N}_{0}$ are unconstrained. Thus the three color conservation constraints (28)-(30) and the color exchange constraint (31) implement the original SU(3) symmetry as a set of constraints that govern the flux of the colors in the dual form of the theory. Identifying these constraints is one of the key goals of this paper.

We stress at this point that the form (28)-(31) of the constraints is overcomplete, since it contains six relations constructed out of the original four relations (24)-(27). However, the overcomplete final form (28)-(31) where the constraints are not all independent better illustrates the complete symmetry among the three colors.

The constraints imply matching conditions for the color flux along the links of the lattice, where originally this flux comes from nonzero cycle occupation numbers $p_{x, \mu \nu}^{a b c d}$. Thus at links where plaquettes touch, the corresponding cycle occupation numbers have to be matched such that constraints are obeyed.

For this matching two cases can be distinguished: The trivial case where cycle occupation numbers that sit on the same plaquette together obey the constraints. Obviously this is only a local contribution and does not contribute to the long range physics.

Relevant for the long range physics and thus the continuum limit are the contributions where neighboring plaquettes share a link such that the cycle occupation numbers at neighboring plaquettes are correlated by the constraints. This gives rise to generalized surfaces which we refer to as "worldsheets". To see the worldsheet nature of nonlocal admissible configurations we start with setting a single cycle occupation number to $p_{x, \mu \nu}^{a b c d}=1$. Clearly this violates the constraints along the four links of the plaquette $(x, \mu \nu)$. If one now takes a neighboring plaquette and selects the corresponding cycle occupation number such that at the joint link the constraints are obeyed, then we have a surface consisting of two plaquettes and the contour of links with violated constraints contains six links. One can keep attaching plaquettes with suitably chosen plaquette occupation numbers to grow the $2 \mathrm{D}$ surface further, and the constraints will always be violated along the boundary of that surface. Thus the only way to obtain a nonlocal configuration of nontrivial cycle occupation numbers, such that all constraints are obeyed, is to create a closed surface. Thus the constraints lead to a structure of closed worldsheets for admissible configurations of cycle occupation numbers. And since the ACCs are already Abelian, the corresponding cycle occupation numbers are additive, and the worldsheet picture also holds for cycle occupation numbers with $\left|p_{x, \mu \nu}^{a b c d}\right|>1$.

Before we come to generalizing the ACC approach to including also fermions, let us briefly summarize the dual worldsheet representation we have constructed for pure $\mathrm{SU}(3)$ lattice gauge theory. The partition function of pure SU(3) lattice gauge theory is exactly rewritten as a sum over configurations of the cycle occupation numbers $p_{x, \mu \nu}^{a b c d} \in \mathbb{Z}$. At each link $(x, \mu)$ the fluxes $J_{x, \mu}^{a b}$ defined in (12) collect the flux of the cycle occupation numbers that connect color $a$ at $x$ to color $b$ at $x+\hat{\mu}$. These fluxes are subject to the color conservation constraints (28)-(30) and the color exchange constraint (31). These constraints restrict the admissible configurations of the cycle occupation numbers, and the long range contributions have the interpretation of worldsheets. 
The configurations of the cycle occupation numbers come with weight factors $W[p]$ that are themselves sums $\sum_{\{l, m, \bar{m}\}}$ over configurations of the auxiliary plaquette variables $l_{x, \mu \nu}^{a b c d}$ and the link-based auxiliary variables $m_{x, \mu}^{a b}$ and $\bar{m}_{x, \mu}^{a b}$. In these sums the constraint (22) restricts the configurations of the $m_{x, \mu}^{a b}$ and $\bar{m}_{x, \mu}^{a b}$ by connecting their differences $j_{x, \mu}^{a b}=m_{x, \mu}^{a b}-\bar{m}_{x, \mu}^{a b}$ to $J_{x, \mu}^{12}$. The contributions to $W_{G}[p]$ collect all terms from expanding the Boltzmann factors, as well as combinatorial factors and the beta functions coming from the Haar measure integration. Note that these contributions come with signs going back to the signs in the parametrization of the SU(3) matrices (8).

All terms in the dual representation are organized with respect to powers of $\beta$, such that the dual form (20), (21), (23) constitutes a strong coupling series. We stress again that in this form of the strong coupling series all expansion coefficients are known in closed form. In the following section we introduce the matter fields and show that the ACC approach can be generalized further to obtain a dual form of full QCD.

\section{QCD AT STRONG COUPLING}

The next step towards a full dual worldline/worldsheet representation of QCD is the generalization of the ACC approach to matter fields. In order to simplify the presentation we start with an intermediate step where we consider the strong coupling limit. In this limit we have $\beta=0$, i.e., the gauge action is absent. Note that in the strong coupling regime a continuum limit cannot be performed. Nevertheless, the strong coupling limit of QCD shares some nonperturbative properties with full QCD, such that it is an interesting toy model per se.

For the discussion of the structure of dual worldline representations, which is the main goal of this paper, also strong coupling QCD is an interesting theory: Integrating out the SU(3) link variables will again lead to constraints for the color fluxes along the links, but in strong coupling QCD these fluxes are generated by the fermions, instead of the cycle occupation numbers of pure gauge theory. We will see that structurally the constraints are the same, but for strong coupling fermion loops the constraints are simpler in their interpretation because of the additional restrictions from the Pauli principle.

For simplicity we will consider the derivation for a theory with only one flavor of staggered quarks, but stress that the generalization to an arbitrary number of flavors is trivial. The fermionic partition function in a background of gauge links is given by

$$
Z_{F}[U]=\int D[\bar{\psi}, \psi] e^{-S_{F}[U, \psi, \bar{\psi}]}
$$

where $\psi_{x}$ and $\bar{\psi}_{x}$ are 3-component vectors of Grassmann numbers assigned to the sites $x$ of our four-dimensional lattice. They obey antiperiodic boundary conditions in the Euclidean time direction $(\nu=4)$ and periodic boundary conditions in space. The integration measure in (32) is a product over Grassmann measures $\int D[\bar{\psi}, \psi]=$ $\prod_{x} \prod_{a=1}^{3} \int d \bar{\psi}_{x}^{a} d \psi_{x}^{a}$. We work with the staggered fermion action given by

$$
\begin{aligned}
S_{F}[U, \psi, \bar{\psi}] & =\sum_{x}\left[m \bar{\psi}_{x} \psi_{x}+\sum_{\nu} \frac{\eta_{x, \nu}}{2}\left(\bar{\psi}_{x} U_{x, \nu} \psi_{x+\hat{\nu}} e^{\mu \delta_{\nu, 4}}-\bar{\psi}_{x+\hat{\nu}} U_{x, \nu}^{\dagger} \psi_{x} e^{-\mu \delta_{\nu, 4}}\right)\right] \\
& =\sum_{x}\left[m \sum_{a=1}^{3} \bar{\psi}_{x}^{a} \psi_{x}^{a}+\sum_{\nu} \frac{\eta_{x, \nu}}{2} \sum_{a, b=1}^{3}\left(\bar{\psi}_{x}^{a} U_{x, \nu}^{a b} \psi_{x+\hat{\nu}}^{b} e^{\mu \delta_{\nu, 4}}-\bar{\psi}_{x+\hat{\nu}}^{b} U_{x, \nu}^{a} \star \psi_{x}^{a} e^{-\mu \delta_{\nu, 4}}\right)\right],
\end{aligned}
$$

where $\eta_{x, 1}=1, \eta_{x, 2}=(-1)^{x_{1}}, \eta_{x, 3}=(-1)^{x_{1}+x_{2}}$ and $\eta_{x, 4}=$ $(-1)^{x_{1}+x_{2}+x_{3}}$ are the staggered sign factors. In (33) we also introduce a chemical potential $\mu$, which gives a different weight to forward and backward hopping in the euclidean time direction. The chemical potential will later be useful to identify the particle number in the dual representation in terms of worldlines. In the strong coupling limit we are considering in this section, the full partition function is obtained by integrating the fermionic partition sum with the product Haar measure of the previous section, i.e., $Z=\int D[U] Z_{F}[U]$.
In the first line of (33) we used matrix-vector notation for color, while in the second line the sums over color indices were written explicitly. Also for the theory with fermions this decomposition of the action is the crucial step towards the dualization. It allows one to completely factorize the Boltzmann weight such that every term in the last line of (33) is a single bilinear in the Grassmann variables, and thus all terms commute with each other.

Using this decomposition of the fermion action we write the partition function as 


$$
\begin{aligned}
& Z_{F}=\int D[\bar{\psi}, \psi] \prod_{x} \prod_{a=1}^{3} e^{-m \bar{\psi}_{x}^{a} \psi_{x}^{a}} \prod_{x, \nu} \prod_{a, b=1}^{3} e^{-\frac{\eta_{x, \nu}, \bar{\psi}_{x}^{a}}{2} U_{x, \nu}^{a b} \psi_{x+\nu}^{b} e^{\mu \delta_{\nu, 4}}} e^{\frac{\eta_{x, \nu}}{2} \bar{\psi}_{x+\nu}^{b} U_{x, \nu}^{a b} \star} \psi_{x}^{a} e^{-\mu \delta_{\nu, 4}} \\
& =\int D[\bar{\psi}, \psi] \prod_{x} \prod_{a=1}^{3} \sum_{s_{x}^{a}=0}^{1}\left(m \psi_{x}^{a} \bar{\psi}_{x}^{a}\right)^{s_{x}^{a}} \prod_{x, \nu} \prod_{a, b=1}^{3} \sum_{k_{x, \nu}^{a b}=0}^{1}\left(\frac{-\eta_{x, \nu}}{2} \bar{\psi}_{x}^{a} U_{x, \nu}^{a b} \psi_{x+\hat{\nu}}^{b} e^{\mu \delta_{\nu, 4}}\right)^{k_{x, \nu}^{a b}} \sum_{\bar{k}_{x, \nu}^{a b}=0}^{1}\left(\frac{\eta_{x, \nu}}{2} \bar{\psi}_{x+\hat{\nu}}^{b} U_{x, \nu}^{a b \star} \psi_{x}^{a} e^{-\mu \delta_{\nu, 4}}\right)^{\bar{k}_{x, \nu}^{a b}} \\
& =\left(\frac{1}{2}\right)^{3 V} \sum_{\{s, k, \bar{k}\}}(2 m)^{\sum_{x, a} s_{x}^{a}} e^{\mu \sum_{x, a b}\left[k_{x, 4}^{a b}-\bar{k}_{x, 4}^{a b}\right]} \prod_{x, \nu} \prod_{a, b}(-1)^{k_{x, \nu}^{a b}}\left(\eta_{x, \nu}\right)^{k_{x, \nu}^{a b}+\bar{k}_{x, \nu}^{a b}}\left(U_{x, \nu}^{a b}\right)^{k_{x, \nu}^{a b}}\left(U_{x, \nu}^{a b \star}\right)^{\bar{k}_{x, \nu}^{a b}} \\
& \times \int D[\bar{\psi}, \psi] \prod_{x, a}\left(\psi_{x}^{a} \bar{\psi}_{x}^{a}\right)^{s_{x}^{a}} \prod_{x, \nu} \prod_{a, b}\left(\bar{\psi}_{x}^{a} \psi_{x+\hat{\nu}}^{b}\right)^{k_{x, \nu}^{a b}}\left(\bar{\psi}_{x+\hat{\nu}}^{b} \psi_{x}^{a}\right)^{\bar{k}_{x, \nu}^{a b}}
\end{aligned}
$$

In the first line we rewrote the Boltzmann weight as a product over local exponential factors, which we then Taylor expanded in the second step. Note that here the Taylor series terminate after the linear term due to the nilpotency of the Grassmann variables. We introduce three types of expansion indices (one for every bilinear of the action) that will be the new dual variables for the fermions: $s_{x}^{a}=0,1$ is the dual variable for expanding the color component $a$ of the mass term contribution at site $x$. $k_{x, L}^{a b}=0,1$ generates the forward hop from color $a$ to color $b$ on the link $(x, \nu)$, and $\bar{k}_{x, \nu}^{a b}=0,1$ the backward hop on the same link. When they assume the nontrivial value 1 , the three types of variables activate the corresponding fermion bilinears: The "monomer" variable $s_{x}^{a}$ activates the mass term component with color $a$. The dual variables $k_{x, \nu}^{a b}$ and $\bar{k}_{x, \nu}^{a b}$ that live on links activate the forward and backward nearest neighbor bilinears $\bar{\psi}_{x}^{a} \psi_{x+\hat{\nu}}^{b}$ and $\bar{\psi}_{x+\hat{\nu}}^{b} \psi_{x}^{a}$ that connect color $a$ and $b$ along the link. We refer to these terms as "Abelian color fluxes".
In Fig. 4 we adapt the graphical representation which we developed for the ACCs now also to the fermionic dual variables $s_{x}^{a}, k_{x, \nu}^{a b}$ and $\bar{k}_{x, \nu}^{a b}$. Again we use a lattice with three layers for the three colors. The monomers that are activated by $s_{x}^{a}$ sit on a single site $x$ and are represented by a circle around the color $a$ they refer to. The "link-fluxes" $k_{x, \nu}^{a b}$ and $\bar{k}_{x, \nu}^{a b}$ connect colors $a$ and $b$ along the link $(x, \nu)$, and we represent them with a forward (backward) oriented arrow that connects the color indices $a$ and $b$.

In the last step of Eq. (34) we have already reorganized the terms: We have collected an overall factor of $(1 / 2)^{3 \mathrm{~V}}$ and introduced the notation $\sum_{\{s, k, \bar{k}\}}$ for the sum over all the possible configurations of the fermion dual variables. Finally we write all factors that do not depend on the Grassmann variables in front of the Grassmann integral. This Grassmann integral in the last line of (34) is either vanishing or \pm 1 , depending on the values of the dual variables $s_{x}^{a}, k_{x, L}^{a b}$ and $\bar{k}_{x, \nu}^{a b}$. In particular, it will be
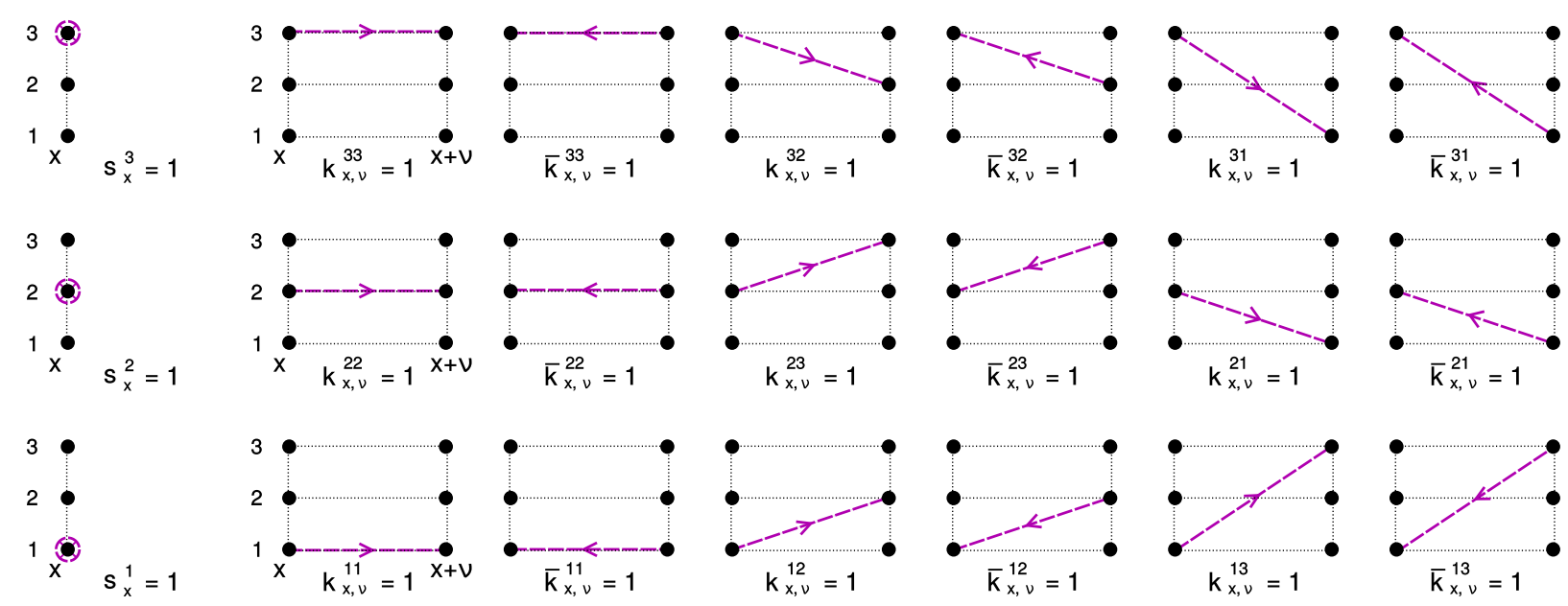

FIG. 4. Graphical representation of the dual variables for the fermions. As before we use a lattice with three layers to represent the color indices. In the first column of diagrams we show the monomers $s_{x}^{a}$, while the arrows in the other columns represent the dual variables $k_{x, L}^{a b}$ and $\bar{k}_{x, \nu}^{a b}$ for the forward and backward hopping, respectively. With these link variables it is possible to build dimers and oriented loops which, together with the monomers, constitute the admissible configurations for fermions. 
nonvanishing only if each Grassmann variable $\psi_{x}^{a} \bar{\psi}_{x}^{a}$ appears exactly once, and we refer to this case as "saturated Grassmann integral".

We may formulate the condition of a saturated Grassmann integral as a constraint for the configurations of the dual variables $s_{x}^{a}, k_{x, \nu}^{a b}$ and $\bar{k}_{x, \nu}^{a b}$, which can be written in the form

$$
\begin{aligned}
C_{F} & {[s, k, \bar{k}] } \\
& =\prod_{x, a} \delta\left(1-s_{x}^{a}-\frac{1}{2} \sum_{\nu, b}\left[k_{x, \nu}^{a b}+\bar{k}_{x, \nu}^{a b}+k_{x-\hat{\nu}, \nu}^{b a}+\bar{k}_{x-\hat{\nu}, \nu}^{b a}\right]\right) .
\end{aligned}
$$

The admissible configurations are known to have a simple structure. Here, for the case where we consider a lattice with three layers, the admissible configurations are such that in all three layers of the four-dimensional lattice each site has to be either occupied by a monomer $\left(s_{x}^{a}=1\right)$, be the end point of a dimer $\left(k_{x, \nu}^{a b}=\bar{k}_{x, \nu}^{a b}=1\right)$, or be run through by a loop $\mathcal{L}$, which is defined as a closed chain of $k_{x, L}^{a b}=1$ and $\bar{k}_{x, \nu}^{a b}=1$.

Having discussed the monomer, dimer and loop structure of admissible fermion configurations we still need to determine the signs of the configurations. Monomers $\psi_{x}^{a} \bar{\psi}_{x}^{a}$ are activated by setting $s_{x}^{a}=1$. It is evident from the last equality in Eq. (34) that monomers simply come with a factor of $2 \mathrm{~m}$, and the Grassmann variables are already in the canonical order we choose for the Grassmann integral $\left(\psi_{x}^{a}\right.$ left of $\left.\bar{\psi}_{x}^{a}\right)$. Thus monomers always contribute to admissible configurations with the explicitly positive factor of $2 m$.

Dimers are constructed by setting $k_{x, L}^{a b}=\bar{k}_{x, L}^{a b}=1$. For the Grassmann integral this corresponds to activating the factor

$$
\bar{\psi}_{x}^{a} \psi_{x+\hat{\nu}}^{b} \bar{\psi}_{x+\hat{\nu}}^{b} \psi_{x}^{a}=-\psi_{x}^{a} \bar{\psi}_{x}^{a} \psi_{x+\hat{\nu}}^{b} \bar{\psi}_{x+\hat{\nu}}^{b} .
$$

The minus sign on the right-hand side of (36) results from the reordering of the Grassmann variables into the canonical order. However, this minus sign is compensated by the explicit minus sign for the forward hop, which in (34) is taken into account in the factor $(-1)^{k_{x, \nu}^{a b}}=(-1)^{1}=-1$. Also the staggered sign factor contribution is always positive for dimers, since $\left(\eta_{x, \nu}\right)_{x, \nu}^{a b+\bar{k}_{x, \nu}^{a b}}=\left(\eta_{x, \nu}\right)^{2}=1$. Finally, also a possible minus sign from the antiperiodic temporal boundary conditions for the fermions is irrelevant since for a dimer such a sign would appear twice. Thus, dimers always come with a positive sign, and only loops can generate negative signs.

The overall sign of a loop receives several contributions. Each loop $\mathcal{L}$ picks up a minus sign from commuting the Grassmann variables into the canonical order. Moreover, each forward hop of the loop will contribute with a minus sign. Hence, if $|\mathcal{L}|$ denotes the length of the loop $\mathcal{L}$, the sign coming from the forward hops is $(-1)^{|\mathcal{L}| / 2}$, since half of the hops in a closed loop are in forward direction. An exception are loops that wind around the compact boundaries, but we may restrict ourselves to choosing lattice extents that are a multiple of 4 , in which case $(-1)^{|\mathcal{L}| 2}$ is correct also for loops that wind. Related to the winding of the loops is also the sign that is generated by the antiperiodic temporal boundary conditions. For every crossing of the last temporal link an additional factor of -1 has to be taken into account, such that the sign factor $(-1)^{W_{\mathcal{L}}}$ emerges, where $W_{\mathcal{L}}$ is the temporal winding number of the loop $\mathcal{L}$.

Finally we have to determine the sign that comes from the staggered sign factors along the links of the loop. Let us first consider a loop around a single plaquette $(x, \rho \nu)$. Around the plaquette the contribution from the staggered signs is given by

$$
\eta_{x, \rho} \eta_{x+\hat{\rho}, \nu} \eta_{x+\hat{\nu}, \rho} \eta_{x, \nu}=-1
$$

and moreover this factor of -1 is independent of the position and orientation of the plaquette. If we then consider two adjacent plaquettes, the staggered sign on the common link will cancel out from the product of staggered factors because it gets squared. Thus the sign of two adjacent plaquettes is also the sign from the staggered factors for the loop along the boundary of the two plaquettes. This mechanism can be iterated to construct a loop of any shape, and the sign coming from the staggered factor can be expressed as $(-1)^{P_{\mathcal{L}}}$, where $P_{\mathcal{L}}$ is the number of plaquettes in the surface bounded by the loop $\mathcal{L}$. Because of the fact that we have three layers of colors, the admissible configurations may also contain loops that wind around the same contour up to 3 times (see Fig. 7 for a simple example of such a loop). For these cases we need a multiply covered surface (e.g., a surface that is covered 3 times for the example in the bottom plot of Fig. 7), and the total number $P_{\mathcal{L}}$ of plaquettes in the surface spanned by the loop is understood in the sense that it also takes into account multiple coverings. We finally remark that the surface that has a loop $\mathcal{L}$ as its boundary is not unique, but it is easy to see that different surfaces with the same boundary differ by an even number of plaquettes, such that the sign factor $(-1)^{P_{\mathcal{L}}}$ remains unchanged.

We can summarize our discussion of the admissible fermion configurations as follows: Admissible configurations are those where every site in our 3-layer lattice is either occupied by a monomer, is the end point of a dimer, or is run through by a loop. Monomers $\left(s_{x}^{a}=1\right)$ come with a factor of $2 m$. Dimers $\left(k_{x, \nu}^{a b}=\bar{k}_{x, \nu}^{a b}=1\right)$ come with a factor of 1 , but also activate the $\mathrm{SU}(3)$ matrix elements along the link, i.e., they activate the factor $U_{x, \nu}^{a b} U_{x, \nu}^{a b}$, that will contribute in the Haar measure integration. Finally loops $\mathcal{L}$ come with a sign factor, which, following the discussion above, is given by 


$$
\operatorname{sign}(\mathcal{L})=(-1)^{1+|\mathcal{L}| / 2+P_{\mathcal{L}}+W_{\mathcal{L}},}
$$

where $|\mathcal{L}|$ is the length of the loop $\mathcal{L}, P_{\mathcal{L}}$ is the number of plaquettes necessary to cover the surface bounded by the loop $\mathcal{L}$, and $W_{\mathcal{L}}$ is the number of temporal windings of $\mathcal{L}$.

To obtain the full strong coupling partition sum $Z=\int D[U] Z_{F}[U]$, we still have to integrate the fermionic partition function $Z_{F}[U]$ over the product of SU(3) Haar measures. We find

$$
\begin{aligned}
Z= & \sum_{\{s, k, \bar{k}\}} C_{F}[s, k, \bar{k}] W_{F}[s, k, \bar{k}] \\
& \times \int D[U] \prod_{x, \nu} \prod_{a, b}\left(U_{x, \nu}^{a b}\right)^{k_{x, \nu}^{a b}}\left(U_{x, \nu}^{a b}\right)^{\bar{k}_{x, \nu}^{a b}}
\end{aligned}
$$

where we introduced the weight for the fermion configurations $W_{F}[s, k, \bar{k}]$ defined as

$$
\begin{aligned}
W_{F} & {[s, k, \bar{k}] } \\
& =\left(\frac{1}{2}\right)^{3 V} \prod_{\mathcal{L}} \operatorname{sign}(\mathcal{L}) \prod_{x}\left[\prod_{a}(2 m)^{s_{x}^{a}}\right]\left[\prod_{a b} e^{\mu\left[k_{x, 4}^{a b}-\bar{k}_{x, 4}^{a b}\right]}\right] \\
& =\left(\frac{1}{2}\right)^{3 V} \prod_{\mathcal{L}} \operatorname{sign}(\mathcal{L}) e^{\mu \beta W_{\mathcal{L}}}\left[\prod_{x, a}(2 m)^{s_{x}^{a}}\right] .
\end{aligned}
$$

In the last step we have simplified the term that couples to the chemical potential $\mu$ : The chemical potential multiplies the difference $k_{x, 4}^{a b}-\bar{k}_{x, 4}^{a b}$ of the temporal forward and backward fluxes. For dimers this difference is zero such that they do not couple to $\mu$. Thus only fermion loops contribute to the $\mu$-dependence. The fermion loops are made from chains of $k_{x, \nu}^{a b}$ and $\bar{k}_{x, \nu}^{a b}$ where at each site the flux is conserved. Consequently only loops that wind around the compact time direction can have nonvanishing $\sum_{x} \sum_{a b}\left(k_{x, 4}^{a b}-\bar{k}_{x, 4}^{a b}\right)$, and it is obvious that for a loop $\mathcal{L}$ this sum is given by $N_{t} W_{\mathcal{L}}$, where $N_{t}$ is the temporal extent of the lattice and $W_{\mathcal{L}}$ is the temporal winding number of the loop $\mathcal{L}$. Using the fact that the inverse temperature $\beta$ in lattice units is given by $N_{t}$ we end up with the expression for the coupling to $\mu$ given in the last line of (40).

Comparing the $\mu$-dependence in the last line of (40) with the usual form $e^{\mu \beta \mathcal{N}}$ for the coupling of the chemical potential, where $\mathcal{N}$ is the net-particle number, we conclude that the net particle number is given by $\mathcal{N}=\sum_{\mathcal{L}} W_{\mathcal{L}}$. Thus we find a nice geometrical interpretation of the net particle number $\mathcal{N}$ in the worldline formulation: $\mathcal{N}$ is given by the total temporal net-winding number of all fermion loops.

We stress that this identification of the net particle number as a topological quantity, i.e., the total temporal net winding number of the loops, is quite different from the manifestation of the particle number in the conventional representation: There the net particle number is given by the discretized integral over the zero component of the conserved vector current, clearly a quantity that is challenging to determine and usually not an integer. In the worldline representation, on the other hand, the temporal winding number is a simple quantity, and it is very easy to define the canonical ensemble by the class of configurations with a fixed temporal net winding number of the fermion loops. We consider this to be one of the most beautiful geometrical aspects of the worldline formulation of QCD. Furthermore, in a toy model it was demonstrated recently that the simple form of the net particle number can be used to implement worldline simulations of the canonical ensemble [27,28].

Having completed the discussion of the fermionic part let us now continue with the remaining gauge integration. The integral over the gauge fields in the last line of (39) can be done in the same way as the corresponding integrals in the pure gauge theory case discussed in the previous section. We insert the path integral measure $D[U]$ and the explicit parametrization (8) for the matrix elements $U_{x, \nu}^{a b}$. For those matrix elements that are sums of complex numbers we use again the binomial representation (15). However, since $k_{x, \nu}^{a b}, \bar{k}_{x, \nu}^{a b}, m_{x, \nu}^{a b}, \bar{m}_{x, \nu}^{a b} \in\{0,1\}$ all binomial factors $\left(\begin{array}{c}k_{x, \mu}^{a b} \\ m_{x, \mu}^{a b}\end{array}\right)$ and $\left(\frac{\bar{k}_{x, \mu}^{a b}}{\bar{m}_{x, \mu}^{a b}}\right)$ are equal to 1 , and we can drop them here. Hence, for the partition function of strong coupling QCD we obtain

$$
Z=\sum_{\{s, k, \bar{k}\}} C_{F}[s, k, \bar{k}] W_{F}[s, k, \bar{k}] C_{G}[k, \bar{k}] W_{G}[k, \bar{k}] .
$$

The gauge field integration in (39) has generated a link based gauge constraint $C_{G}[k, \bar{k}]$ and a gauge field weight factor $W_{G}[k, \bar{k}]$. To represent the constraints and the weight factor in a transparent way, we introduce combinations of the dual variables $k_{x, \nu}^{a b}, \bar{k}_{x, \nu}^{a b}$ and the auxiliary variables $m_{x, \nu}^{a b}$, $\bar{m}_{x, \nu}^{a b}$ for $(a, b)=(2,1),(2,3),(3,1),(3,3)$ as follows:

$$
\begin{aligned}
K_{x, \nu}^{a b} & =k_{x, \nu}^{a b}-\bar{k}_{x, \nu}^{a b}, \\
j_{x, \nu}^{a b} & =m_{x, \nu}^{a b}-\bar{m}_{x, \nu}^{a b}, \quad P_{x, \nu}^{a b}=k_{x, \nu}^{a b}+\bar{k}_{x, \nu}^{a b}, \\
a b & =m_{x, \nu}^{a b}+\bar{m}_{x, \nu}^{a b} .
\end{aligned}
$$

Again constraints are generated by the integration over the phases $\phi_{x, \nu}^{(j)}$ of the representation (8), and as in the pure gauge case we can organize them such that four of them give rise to relations among the fluxes $K_{x, \nu}^{a b}$. These gauge field constraints are denoted by $C_{G}[k, \bar{k}]$ in (41) and are explicitly given by

$$
\begin{aligned}
C_{G}[k, \bar{k}]= & \prod_{x, \nu} \delta\left(K_{x, \mu}^{12}+K_{x, \nu}^{13}-K_{x, \nu}^{21}-K_{x, \nu}^{31}\right) \\
& \times \delta\left(K_{x, \nu}^{21}+K_{x, \nu}^{23}-K_{x, \nu}^{12}-K_{x, \nu}^{32}\right) \\
& \times \delta\left(K_{x, \nu}^{11}+K_{x, \nu}^{12}-K_{x, \nu}^{23}-K_{x, \nu}^{33}\right) \\
& \times \delta\left(K_{x, \nu}^{31}+K_{x, \nu}^{33}-K_{x, \nu}^{12}-K_{x, \nu}^{22}\right) .
\end{aligned}
$$


The weight factor $W_{G}[k, \bar{k}]$ is given as a sum over configurations of the $m_{x, \nu}^{a b}, \bar{m}_{x, \nu}^{a b}$ and contains another constraint,

$$
K_{x, \nu}^{12}=j_{x, \nu}^{21}+j_{x, \nu}^{23}+j_{x, \nu}^{31}+j_{x, \nu}^{33}
$$

which comes from integrating over $\phi_{x, \nu}^{(3)}$ and connects the sum of auxiliary variables $j_{x, \nu}^{a b}$ to $K_{x, \nu}^{12}$. Explicitly the weight factor is given by

$$
\begin{aligned}
W_{G}[k, \bar{k}]= & 2^{4 V} \sum_{\{m, \bar{m}\}}\left[\prod_{x, \nu} \delta\left(K_{x, \nu}^{12}-j_{x, \nu}^{21}-j_{x, \nu}^{23}-j_{x, \nu}^{31}-j_{x, \nu}^{33}\right)\right]\left[\prod_{x, \nu}(-1)^{\left.K_{x, \nu}^{12}+K_{x, \nu}^{23}+K_{x, \nu}^{31}-j_{x, \nu}^{23}-j_{x, \nu}^{31}\right]}\right. \\
& \times\left[\prod_{x, \nu} B\left(\frac{P_{x, \nu}^{11}+P_{x, \nu}^{13}+P_{x, \nu}^{22}+P_{x, \nu}^{32}}{2}+2 \mid \frac{P_{x, \nu}^{12}+s_{x, \nu}^{21}+s_{x, \nu}^{23}+s_{x, \nu}^{31}+s_{x, \nu}^{33}}{2}+1\right)\right. \\
& \times B\left(\frac{P_{x, \nu}^{11}+s_{x, \nu}^{21}+P_{x, \nu}^{23}-s_{x, \nu}^{23}+s_{x, \nu}^{31}+P_{x, \nu}^{33}-s_{x, \nu}^{33}}{2}+1 \mid \frac{P_{x, \nu}^{13}+P_{x, \nu}^{21}-s_{x, \nu}^{21}+s_{x, \nu}^{23}+P_{x, \nu}^{31}-s_{x, \nu}^{31}+s_{x, \nu}^{33}}{2}+1\right) \\
& \left.\times B\left(\frac{s_{x, \nu}^{21}+P_{x, \nu}^{22}+s_{x, \nu}^{23}+P_{x, \nu}^{31}-s_{x, \nu}^{31}+P_{x, \nu}^{33}-s_{x, \nu}^{33}}{2}+1 \mid \frac{P_{x, \nu}^{21}-s_{x, \nu}^{21}+P_{x, \nu}^{23}-s_{x, \nu}^{23}+s_{x, \nu}^{31}+P_{x, \nu}^{32}+s_{x, \nu}^{33}}{2}+1\right)\right],
\end{aligned}
$$

where we have defined

$$
\sum_{\{m, \bar{m}\}}=\prod_{x, \nu} \prod_{a=2,3} \prod_{b=1,3} \sum_{m_{x, \nu}^{a b}=0}^{k_{x, \nu}^{a b}} \sum_{\bar{m}_{x, \nu}^{a b}=0}^{\bar{k}_{x, \nu}^{a b}} .
$$

Having completed the derivation of the dual representation for strong coupling QCD collected in Eqs. (41), (43) and (45), it is highly instructive to discuss the structural similarity with the dual representation of the pure gauge theory case in Eqs. (20), (21) and (23).

In both, the pure gauge theory and the strong coupling QCD cases, we have color flux that lives on the links of the lattice and connects the three different color labels on both ends of the link. In pure gauge theory this flux is generated by the plaquette-based cycle occupation numbers $p_{x, \mu \nu}^{a b c d}$, which contribute to the fluxes on all four links of the plaquette. Consequently the pure gauge theory partition sum (20) is a sum over all configurations of the cycle occupation numbers. In the strong coupling QCD case the color flux on the links is generated by fermion loops. These fermion loops are described by the dual fermion variables $k_{x, \mu}^{a b}$ and $\bar{k}_{x, \mu}^{a b}$, which together with the monomer variables $s_{x}^{a}$ have to obey the fermion constraints $C_{F}[s, k, \bar{k}]$ in (35). The fermion constraints force the variables $k_{x, \mu}^{a b}$ and $\bar{k}_{x, \mu}^{a b}$ to form closed loops of color flux. These fluxes around closed loops may be viewed as generalizations of the fluxes in pure gauge theory which generated by nonzero cycle occupation numbers $p_{x, \mu \nu}^{a b c d}$ and thus are only around single plaquettes.

Having understood that in both cases we deal with link based fluxes around closed loops (plaquettes or general loops), we can now compare the gauge field weight factors and the constraints. A comparison of the weight factor
$W_{G}[p]$ of the pure gauge theory in Eq. (23) and the weight factor $W_{G}[k, \bar{k}]$ for strong coupling QCD in (45) shows their structural similarity. Both are sums over configurations of the auxiliary variables $m_{x, \nu}^{a b}$ and $\bar{m}_{x, \nu}^{a b}$ needed for the binomial decomposition. In both cases the same auxiliary constraint connects the configurations of these via the combination $j_{x, \nu}^{a b}=m_{x, \nu}^{a b}-\bar{m}_{x, \nu}^{a b}$ to the $K_{x, \nu}^{12}$ color flux at every link. Furthermore, the same sign factors appear in the summands of both weight factors. Because of the Pauli principle, the fluxes in the strong coupling case are restricted to the values 0,1 and -1 , such that all binomial coefficients are equal to 1 , while in the pure gauge theory weight $W_{G}[p]$ in Eq. (23) the binomial coefficients can have nontrivial values. Furthermore, in the pure gauge theory weight $W_{G}[p]$ we have plaquette based weight factors from the expansion of the gauge action which also depend on the auxiliary plaquette variables $l_{x, \mu \nu}^{a b c d}$. Clearly these terms are absent in strong coupling QCD where we have no gauge action.

However, the weight factors that come from the Haar measure integration, and thus (together with the constraints) are responsible for implementing the $\mathrm{SU}(3)$ symmetry in the worldline representation, are identical in the two cases: They are given as the product of the three beta functions that appear in (23) and in (45) and come from integrating the three angles $\theta_{x, \nu}^{(j)}$ with the corresponding Haar measure contributions. Obviously, in (23) and in (45) these weights also couple to the same color flux components.

Also the second ingredient that is necessary to implement the SU(3) symmetry of the conventional representation, i.e., the constraints, are the same for the pure gauge theory and strong coupling QCD. From (43) we read off the relations 


$$
\begin{aligned}
& K_{x, \nu}^{12}+K_{x, \nu}^{13}=K_{x, \nu}^{21}+K_{x, \nu}^{31}, \\
& K_{x, \nu}^{21}+K_{x, \nu}^{23}=K_{x, \nu}^{12}+K_{x, \nu}^{32} \\
& K_{x, \nu}^{11}+K_{x, \nu}^{12}=K_{x, \nu}^{23}+K_{x, \nu}^{33}, \\
& K_{x, \nu}^{31}+K_{x, \nu}^{33}=K_{x, \nu}^{12}+K_{x, \nu}^{22},
\end{aligned}
$$

which are structurally identical to those for the fluxes $J_{x, \nu}^{a b}$ of pure gauge theory in (24)-(27). Thus we can recombine them in the same way and bring them to the form of (28)(31), giving rise to the same geometrical interpretation which we illustrated in Fig. 3.

The structural similarity for the constraints and the weights we have discussed constitutes the essence of the dual worldline/worldsheet representation for systems with $\mathrm{SU}(3)$ gauge fields. Other aspects, such as implications of the constraints for the matter field worldlines are specific for the type of matter the gauge links couple to. Let us now address this aspect in more detail for the case of strong coupling QCD and discuss the structure of strong coupling fermion loops.

\section{STRONG COUPLING BARYON FLUXES}

For the case of strong coupling QCD, only the dual fermion variables $k_{x, \nu}^{a b}$ and $\bar{k}_{x, \nu}^{a b}$ generate color flux. Since these variables can only be 0 or 1 , the corresponding color flux variables $K_{x, \nu}^{a b}=k_{x, \nu}^{a b}-\bar{k}_{x, \nu}^{a b}$ that enter the constraints (47)-(50) are restricted to the values $-1,0,1$, where $K_{x, \nu}^{a b}=$ +1 corresponds to a unit flux from color $a$ at $x$ into color $b$ at $x+\hat{\nu}$ and $K_{x, \nu}^{a b}=-1$ to the corresponding flux in the opposite direction. Thus we have only a small number of possible color flux configurations on a link $(x, \nu)$ which are further restricted by the constraints in Eqs. (47)-(50).

Also the auxiliary variables $m_{x, L}^{a b}$ and $\bar{m}_{x, \nu}^{a b}$ for the binomial decomposition which we sum over in the gauge field weight $W_{G}[k, \bar{k}]$ in (45) are highly restricted since $0 \leq m_{x, \nu}^{a b} \leq k_{x, \nu}^{a b} \leq 1$ and $0 \leq \bar{m}_{x, \nu}^{a b} \leq \bar{k}_{x, \nu}^{a b} \leq 1$. Furthermore, via $j_{x, \nu}^{a b}=m_{x, \nu}^{a b}-\bar{m}_{x, \nu}^{a b}$ they are restricted further by the constraint

$$
j_{x, \nu}^{21}+j_{x, \nu}^{23}+j_{x, \nu}^{31}+j_{x, \nu}^{33}=K_{x, \nu}^{12},
$$

that appears in the gauge field weight $W_{G}[k, \bar{k}]$ in (45). Since both, the dual fermion variables $k_{x, \nu}^{a b}, \bar{k}_{x, \nu}^{a b}$, as well as the auxiliary variables $m_{x, \nu}^{a b}, \bar{m}_{x, \nu}^{a b}$ are highly restricted in strong coupling QCD, we can completely list all flux combinations that are admissible at a given link. In addition we can determine the corresponding sign that appears in the weight $W_{G}[k, \bar{k}]$, which for a link $(x, \nu)$ is given by

$$
(-1)^{K_{x, \nu}^{12}+K_{x, \nu}^{23}+K_{x, \nu}^{31}-j_{x, \nu}^{23}-j_{x, \nu}^{31} .}
$$

The admissible combinations of the strong coupling fluxes $K_{x, \nu}^{a b}$ at a single link come in two types: Three lines of flux that run in the same direction (see Fig. 5), or six lines of flux that form a closed loop on a single link (Fig. 6). Obviously only the first type allows for long distance propagation and we refer to these strong coupling elements as "strong coupling baryon fluxes". The locally closing ones are referred to as "one link loops".

For the discussion of the complete list of strong coupling baryon fluxes we start with solutions of the constraint equations (47)-(50) where we allow only the values $K_{x, \nu}^{a b}=1$, 0, i.e., we consider forward propagation. In addition to the gauge constraints also the fermion constraints have to be obeyed, which imply that from a node with fixed space time $x$ and fixed color $a$ only a single forward arrow may origin. One finds exactly six solutions, and Fig. 5 shows the admissible strong coupling baryon fluxes for forward propagation. The six strong coupling baryon fluxes for backward propagation are obtained by reverting the arrows, which corresponds to $K_{x, \nu}^{a b} \rightarrow-K_{x, \nu}^{a b}$.

The signs (52) are easy to determine for these six configurations. Let us discuss two examples: For the top left example in Fig. 5 we have the nonvanishing fluxes $K_{x, \nu}^{11}=K_{x, \nu}^{22}=K_{x, \nu}^{33}=1$. Since $K_{x, \nu}^{23}=K_{x, \nu}^{31}=0$, also $j_{x, \nu}^{23}$ and $j_{x, \nu}^{31}$ must vanish, such that the sign (52) is +1 .

The top center example in Fig. 5 has the nonvanishing fluxes $K_{x, \nu}^{12}=K_{x, \nu}^{23}=K_{x, \nu}^{31}=1$, and these three terms alone give a minus sign in (52). However, since $K_{x, \nu}^{12}=1$ we must also have one of the $j_{x, \nu}^{21}, j_{x, \nu}^{23}, j_{x, \nu}^{31}$, or $j_{x, \nu}^{33}$ to be set to 1 , in order to obey the additional constraint (51). Since only $K_{x, \nu}^{23}$ and $K_{x, \nu}^{31}$ are nonzero, either $j_{x, \nu}^{23}$ or $j_{x, \nu}^{31}$ must be 1 , and either choice brings the total sign in (52) back to +1 .

In a similar way one may analyze the sign for all strong coupling baryon fluxes, and one finds the simple result

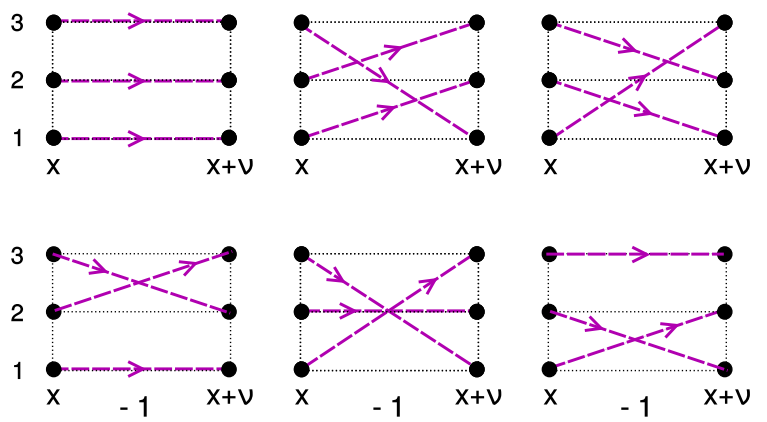

FIG. 5. Baryon loop elements in the strong coupling limit. Only the six combinations shown here are admissible and propagating fluxes in the strong coupling limit. The elements with an odd number of color flux crossings come with an explicit minus sign. For the negative direction the same fluxes are admissible and have the same signs. The corresponding diagrams are obtained by reverting the arrows. 

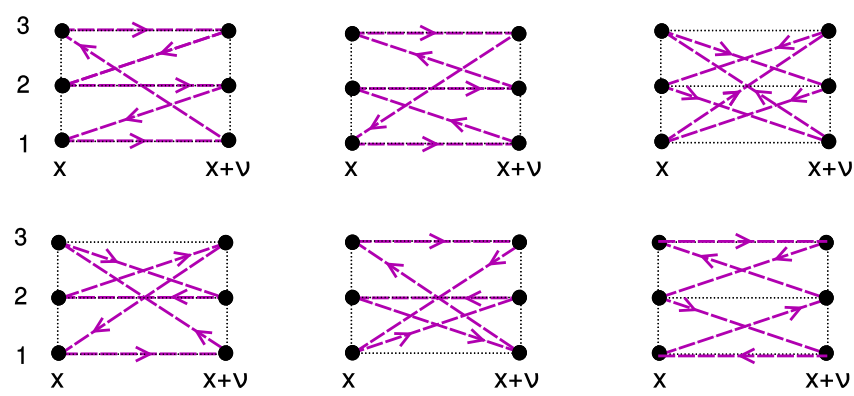

FIG. 6. Closed, nonpropagating one-link loops at strong coupling. All of these loops come with a positive weight. Also the opposite orientation is possible, which is obtained by reverting all arrows.

$$
(-1)^{K_{x, \nu}^{12}+K_{x, \nu}^{23}+K_{x, \nu}^{31}-j_{x, \nu}^{23}-j_{x, \nu}^{31}}=(-1)^{\text {crossings of } K \text {-flux }} .
$$

In Fig. 5 the strong coupling baryon fluxes where this sign is negative are marked with -1 . In addition one may evaluate the weight given by the product of beta functions in (45), and a simple calculation shows that this weight has the value of 1/12 for all six strong coupling baryon fluxes shown in Fig. 5.

The second class of solutions of (47)-(50), i.e., the one link loops are obtained by now allowing all values $K_{x, \nu}^{a b}=-1,0,+1$ and enforcing the fermion constraints, such that each node is run through by a loop. The corresponding solutions are depicted in Fig. 6, and it is easy to see that they are obtained by combining one of the forward baryon fluxes from Fig. 5 with a matching backward baryon flux such that the fermion constraints are obeyed. One finds that only the fluxes with the same sign in Fig. 5 can be combined among each other, such that the total sign from (52) is always +1 . According to (38) the emerging loops also have a positive fermion loop sign: There is an overall minus sign and a factor $(-1)^{3}$ for the three forward hops. Thus one link loops always come with a positive weight which is given by the products of beta functions in (45). These weights can be summed, and all possible one link loops may be combined into a dual element that plays a similar role as the monomers and dimers: They are all local fermionic monomials that can be used to saturate the fermion constraints on the sites not occupied by a strong coupling baryon loop.

We now conclude the discussion of strong coupling QCD, by showing that the loop signs (38), the gauge signs (52) and the constraints (47)-(50) conspire in such a way, that the remaining strong coupling baryon loops again obey the sign formula (38) for staggered fermions.

For this proof we start with a strong coupling baryon loop made out of only the top left flux elements of Fig. 5, i.e., only flux elements with parallel fluxes for all three colors are used. A very simple example of such a loop around a single plaquette is shown in the top plot of Fig. 7. Obviously such a loop is made out of three copies of the
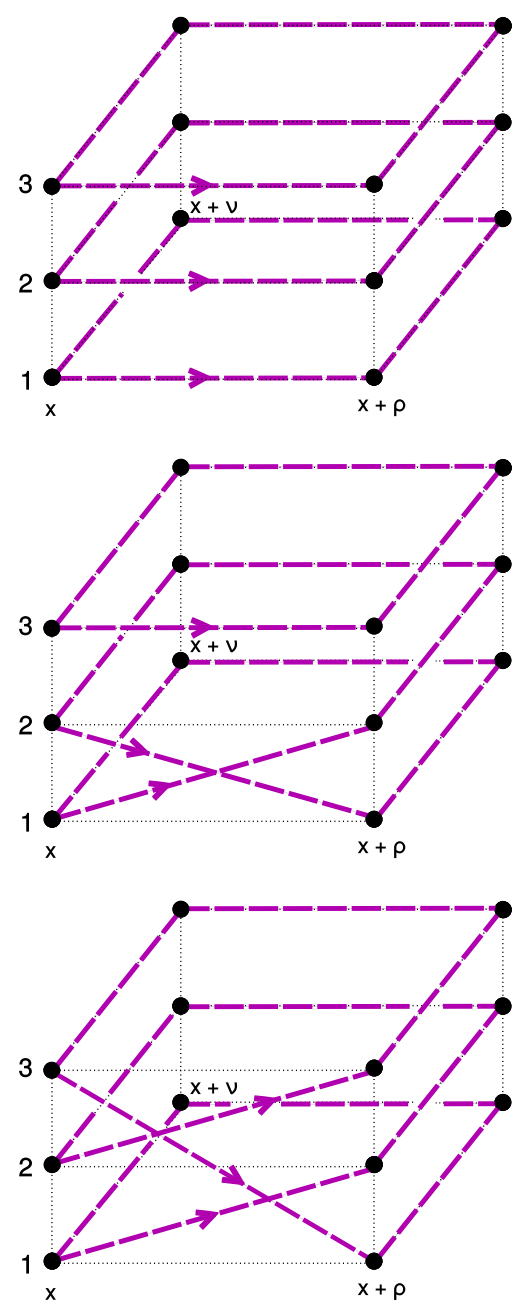

FIG. 7. Examples of simple strong coupling baryon loops with different connectivity properties.

same quark loop. Each one of these loops $\mathcal{L}$ has the sign factor $\operatorname{sign}(\mathcal{L})$ for staggered fermion loops as given in (38). Thus for the strong coupling baryon loop made out of only the top left flux combinations in Fig. 5, we find the sign

$$
\operatorname{sign}(\mathcal{L})^{3}=\operatorname{sign}(\mathcal{L})
$$

Note that this identity also holds for temporally winding loops where an additional sign is picked up from the antiperiodic boundary conditions. Now we can replace the top left flux elements of Fig. 5 by one of the other strong coupling baryon flux elements where color fluxes cross. An example with an element with one crossing is shown in the middle plot of Fig. 7, and in the bottom plot we have replaced one of the parallel elements with a strong coupling baryon flux with two crossings. However, every crossing of flux also changes the connectivity properties of the loop: Inserting one crossing either connects two fermion loops into one, see, e.g., the example in the middle of Fig. 7, or splits a loop into two components. Thus every crossing changes the number of loops by one, and since every loop 
comes with an overall minus sign, inserting one crossing changes the fermion sign. However, we have shown that the gauge sign in Eq. (53) changes with the number of crossings, such that the gauge sign and the fermion sign cancel. Consequently the sign of the baryon loops is always given by (54), i.e., the signs of the strong coupling baryon loops are the signs for loops of a single free staggered fermion.

We can make the identification of the strong coupling baryon loops with the loops of a free staggered fermion complete by using the fact that all the strong coupling flux elements in Fig. 5 come with the same weight 1/12. Thus at every link of the loop we can sum over all six possible fluxes and obtain a total link weight of $1 / 2$. We conclude that the dual form of strong coupling QCD is a gas of free staggered fermion loops with a link weight of $1 / 2$. These loops describe baryons and are embedded in a background of monomers, dimers and local link loops, such that the fermion constraints are obeyed.

We are currently exploring the possibility of updating our form of strong coupling QCD with fermion bags [29-31]: One can sum up the weights of all local link loops in Fig. 6 and all combinations of three dimers that saturate the fermion constraints on a single link. All these terms give rise to an effective baryon dimer with a weight larger than $1 / 4$, which is the weight of a dimer from staggered fermions with a link factor $1 / 2$. Splitting the overall weight of the effective baryon dimer in the form $1 / 4+g$, we may treat the part with a factor $g$ as an interaction for the free staggered fermions used for the baryons, together with the remaining configurations not yet taken into account, i.e., mixed contributions of monomers and dimers on a link and closed chains of single and double dimer links. These interaction terms come with positive factors and can be activated according to their weight, such that activated terms delimit the fermion bags inside which the free staggered fermions for the baryons may propagate.

\section{FULL QCD}

We complete the presentation of the dual representation in terms of worldlines and worldsheets with discussing the case of full QCD. The partition function of full QCD can be written as

$$
Z=\int D[U] Z_{F}[U] e^{-S_{G}[U]},
$$

i.e., the fermionic partition function $Z_{F}[U]$ given in Eq. (32) is now integrated over with the Boltzmann factor for the gauge action $S_{G}[U]$ (1).

In Sec. III we have obtained the intermediate result (39) where the fermionic partition function $Z_{F}[U]$ in a fixed gauge background is already expressed as a sum over configurations of the dual fermion variables $s_{x}^{a}, k_{x, \nu}^{a b}$ and $\bar{k}_{x, \nu}^{a b}$. The dual variables $k_{x, \nu}^{a b}$ and $\bar{k}_{x, \nu}^{a b}$ for fermion hopping activate the corresponding link matrix elements $U_{x, \nu}^{a b}$ and
$U_{x, \nu}^{a b \star}$ which in the strong coupling expression (39) simply were integrated over with the gauge field measure $\int D[U]=\prod_{x, \nu} \int d U_{x, \nu}$.

In full QCD the gauge field integral now also has to take into account the gauge field Boltzmann factor, such that the resulting integral reads

$$
\left[\prod_{x, \nu} \int d U_{x, \nu}\right] e^{-S_{G}[U]} \prod_{x, \nu} \prod_{a, b}\left(U_{x, \nu}^{a b}\right)^{k_{x, \nu}^{a b}}\left(U_{x, \nu}^{a b \star}\right)^{\bar{k}_{x, \nu}^{a b}} .
$$

The Boltzmann factor $e^{-S_{G}[U]}$ can again be treated as in Sec. II, i.e., we expand in Abelian color cycles and organize the terms with respect to the links $(x, \nu)$ and color indices $a, b$. Thus the remaining gauge field integral reads

$$
\left[\prod_{x, \nu} \int d U_{x, \nu}\right] \prod_{a, b}\left(U_{x, \nu}^{a b}\right)^{N_{x, \nu}^{a b}+k_{x, \nu}^{a b}}\left(U_{x, \nu}^{a b \star}\right)^{\bar{N}_{x, \nu}^{a b}+\bar{k}_{x, \nu}^{a b}},
$$

where $N_{x, \nu}^{a b}$ and $\bar{N}_{x, \nu}^{a b}$ are the same combinations as defined in (14). This is the same integral as in the intermediate result (5), only the $N_{x, \nu}^{a b}$ and $\bar{N}_{x, \nu}^{a b}$ are now replaced by $N_{x, \nu}^{a b}+k_{x, \nu}^{a b}$ and $\bar{N}_{x, \nu}^{a b}+\bar{k}_{x, \nu}^{a b}$. Consequently we can simply follow the steps in Sec. II. We again write the exponents $U_{x, L}^{a b}$ and $U_{x, L}^{a b}$ in the form

$N_{x, \mu}^{a b}+k_{x, \mu}^{a b}=\frac{Q_{x, \mu}^{a b}+L_{x, \mu}^{a b}}{2}, \quad \bar{N}_{x, \mu}^{a b}+\bar{k}_{x, \mu}^{a b}=\frac{Q_{x, \mu}^{a b}-L_{x, \mu}^{a b}}{2}$,

where

$$
L_{x, \mu}^{a b}=J_{x, \mu}^{a b}+K_{x, \mu}^{a b}, \quad Q_{x, \mu}^{a b}=S_{x, \mu}^{a b}+P_{x, \mu}^{a b},
$$

where $J_{x, \mu}^{a b}$ and $S_{x, \mu}^{a b}$ defined in (12) and (13) collect the fluxes and weight arguments for the gauge fields, and $K_{x, \mu}^{a b}$ and $P_{x, \mu}^{a b}$ defined in (42) those for the fermions. Again we use $m_{x, \nu}^{a b}$ and $\bar{m}_{x, \nu}^{a b}$ with $(a, b)=(2,1),(2,3),(3,1),(3,3)$ as the auxiliary variables for the binomial decomposition, which now run from 0 to $N_{x, \nu}^{a b}+k_{x, \nu}^{a b}$ and $\bar{N}_{x, \nu}^{a b}+\bar{k}_{x, \nu}^{a b}$, respectively.

Putting things together we find that the dual form of the partition function of full QCD is a sum over configurations $\sum_{\{s, k, \bar{k}, p\}}$ of the fermion dual variables $s_{x}^{a}, k_{x, \nu}^{a b}, \bar{k}_{x, \nu}^{a b} \in\{0,1\}$, as well as the cycle occupation numbers $p_{x, \mu \nu}^{a b c d} \in \mathbb{Z}$,

$Z=\sum_{\{s, k, \bar{k}, p\}} C_{F}[s, k, \bar{k}] W_{F}[s, k, \bar{k}] C_{G}[k, \bar{k}, p] W_{G}[k, \bar{k}, p]$.

The fermion constraint $C_{F}[s, k, \bar{k}]$ is again given by (35) i.e., the admissible worldline configurations are such that every site of the 3-layer lattice is either occupied by a monomer, is the end point of a dimer or is run through by a 
loop $\mathcal{L}$. Also the fermion weights $W_{F}[s, k, \bar{k}]$ are the ones already discussed in (40), i.e., monomers contribute a factor of $2 m$, loops come with a sign $\operatorname{sign}(\mathcal{L})$ given in (38) and the chemical potential couples to the temporal winding number $W_{\mathcal{L}}$ of the loops.

The gauge constraints $C_{G}[k, \bar{k}, p]$ are given by

$$
\begin{aligned}
C_{G}[k, \bar{k}, p]= & \prod_{x, \mu} \delta\left(L_{x, \mu}^{12}+L_{x, \mu}^{13}-L_{x, \mu}^{21}-L_{x, \mu}^{31}\right) \\
& \times \delta\left(L_{x, \mu}^{21}+L_{x, \mu}^{23}-L_{x, \mu}^{12}-L_{x, \mu}^{32}\right) \\
& \times \delta\left(L_{x, \mu}^{11}+L_{x, \mu}^{12}-L_{x, \mu}^{23}-L_{x, \mu}^{33}\right) \\
& \times \delta\left(L_{x, \mu}^{31}+L_{x, \mu}^{33}-L_{x, \mu}^{12}-L_{x, \mu}^{22}\right) .
\end{aligned}
$$

Structurally these are of course the same constraints as for pure gauge theory and strong coupling QCDafter all they are generated by integrating the SU(3) link matrices-but here in full QCD they link the color flux contributions from both, the gauge fields via the cycle occupation numbers $p_{x, \mu \nu}^{a b c d}$ and the fermion loops via $k_{x, L}^{a b}$ and $\bar{k}_{x, \nu}^{a b}$.

The gauge field weights are structurally identical to those of pure gauge theory, but again also the fluxes from the fermions contribute through the combined variables $Q_{x, \nu}^{a b}$. The weights are again a sum $\sum_{\{l, m, \bar{m}\}}$ over configurations of the auxiliary plaquette variables $l_{x, \mu \nu}^{a b c d} \in \mathbb{N}_{0}$ and the auxiliary binomial variables $m_{x, \nu}^{a b}$ and $\bar{m}_{x, \nu}^{a b}$,

$$
\begin{aligned}
& W_{G}[k, \bar{k}, p]=2^{4 V} \sum_{\{l, m, \bar{m}\}}\left[\prod_{x, \mu} \delta\left(L_{x, \mu}^{12}-j_{x, \mu}^{21}-j_{x, \mu}^{23}-j_{x, \mu}^{31}-j_{x, \mu}^{33}\right)\right]\left[\prod_{x, \mu}(-1)^{L_{x, \mu}^{12}+L_{x, \mu}^{23}+L_{x, \mu}^{31}-j_{x, \mu}^{23}-j_{x, \mu}^{31}}\right] \\
& \times\left[\prod_{x, \mu} \prod_{a=2,3} \prod_{b=1,3}\left(\begin{array}{c}
N_{x, \mu}^{a b}+k_{x, \mu}^{a b} \\
m_{x, \mu}^{a b}
\end{array}\right)\left(\begin{array}{c}
\bar{N}_{x, \mu}^{a b}+\bar{k}_{x, \mu}^{a b} \\
\bar{m}_{x, \mu}^{a b}
\end{array}\right)\right]\left[\prod_{x, \mu<\nu} \prod_{a, b, c, d} \frac{(\beta / 2)^{\left|p_{x, \mu \nu}^{a b c d}\right|+2 l_{x, \mu \nu}^{a b c d}}}{\left(\left|p_{x, \mu \nu}^{a b c d}\right|+l_{x, \mu \nu}^{a b c d}\right) ! l_{x, \mu \nu}^{a b c d !} !}\right] \\
& \times\left[\prod_{x, \mu} B\left(\frac{Q_{x, \mu}^{11}+Q_{x, \mu}^{13}+Q_{x, \mu}^{22}+Q_{x, \mu}^{32}}{2}+2 \mid \frac{Q_{x, \mu}^{12}+s_{x, \mu}^{21}+s_{x, \mu}^{23}+s_{x, \mu}^{31}+s_{x, \mu}^{33}}{2}+1\right)\right. \\
& \times B\left(\frac{Q_{x, \mu}^{11}+s_{x, \mu}^{21}+Q_{x, \mu}^{23}-s_{x, \mu}^{23}+s_{x, \mu}^{31}+Q_{x, \mu}^{33}-s_{x, \mu}^{33}}{2}+1 \mid \frac{Q_{x, \mu}^{13}+Q_{x, \mu}^{21}-s_{x, \mu}^{21}+s_{x, \mu}^{23}+Q_{x, \mu}^{31}-s_{x, \mu}^{31}+s_{x, \mu}^{33}}{2}+1\right) \\
& \left.\times B\left(\frac{s_{x, \mu}^{21}+Q_{x, \mu}^{22}+s_{x, \mu}^{23}+Q_{x, \mu}^{31}-s_{x, \mu}^{31}+Q_{x, \mu}^{33}-s_{x, \mu}^{33}}{2}+1 \mid \frac{Q_{x, \mu}^{21}-s_{x, \mu}^{21}+Q_{x, \mu}^{23}-s_{x, \mu}^{23}+s_{x, \mu}^{31}+Q_{x, \mu}^{32}+s_{x, \mu}^{33}}{2}+1\right)\right],
\end{aligned}
$$

where we again used the abbreviations $j_{x, \mu}^{a b} \equiv m_{x, \mu}^{a b}-\bar{m}_{x, \mu}^{a b}$ and $s_{x, \mu}^{a b} \equiv m_{x, \mu}^{a b}+\bar{m}_{x, \mu}^{a b}$ from Eq. (15).

We conclude this section on full QCD with addressing two important aspects of the new representation: As in the case of pure SU(3) lattice gauge theory, our dual form of the partition sum has the structure of a strong coupling expansion, and again, our approach allows one to compute all coefficients of this expansion in closed form.

Furthermore, it is obvious how to generalize the construction to several flavors: One simply uses multiple sets of dual fermion variables, which all couple in the same way to the gauge fields. Thus instead of the variables $k_{x, \nu}^{a b}$ and $\bar{k}_{x, \nu}^{a b}$ one has flavor sums over such variables, and the color fluxes at each link have contributions from all flavors. These flavor sums over the dual fermion variables enter the constraints and weights, which otherwise have the same form as presented in this section.

\section{SUMMARY AND CONCLUSIONS}

In this paper we have presented a new dual worldline/ worldsheet representation of lattice QCD based on the
Abelian color flux approach, where both, the fermion as well as the gauge action are decomposed into minimal terms, the Abelian color fluxes, that connect different color indices at neighboring sites. After expanding the corresponding Boltzmann factors the contributions are organized according to links and the non-Abelian gauge field integrals can be solved in closed form. These integrals lead to weight factors for the fluxes, as well as to constraints sitting on the links.

The approach here is presented for three cases: Pure SU(3) lattice gauge theory, strong coupling lattice QCD and full lattice QCD (the latter two for one flavor of staggered fermions). In the pure $\mathrm{SU}(3)$ case the Abelian color fluxes are generated from Abelian color cycles, which are loops in color space closing around plaquettes. The constraints restrict the possible configurations of Abelian color cycles, and we show that the degrees of freedom responsible for long distance physics are closed worldsheets living on a space-time lattice with three layers for the three colors.

One of the key results is the identification of the constraints of the color fluxes, which are collected in 
Eqs. (28)-(31) and illustrated in Fig. 3. The constraints Eqs. (28)-(30) enforce the individual conservation of flux for all three colors (color conservation constraints), while the constraints (31) ensure the equal distribution of flux among the colors (color exchange constraints). All constraints are structurally the same for the three systems studied here, i.e., pure SU(3) gauge theory, strong coupling QCD and full QCD, although the sources of color flux are different: Plaquette based cycle occupation numbers for the worldsheets of gauge degrees of freedom and link based color fluxes for the fermion worldlines. However, in both cases the constraints (28)-(31) are the dual manifestation of the original $\mathrm{SU}(3)$ symmetry of the conventional representation. We remark again that the dual representation has weight factors with negative signs, and a Monte Carlo simulation might be possible only after finding a suitable resummation scheme.

In strong coupling QCD the Abelian color fluxes are generated by loops and dimers of fermions, which together with monomers for the mass terms constitute the admissible configurations for the fermions. Again the gauge link integrals generate constraints for the color fluxes which are structurally identical to the ones in pure $\mathrm{SU}(3)$ gauge theory. Combining the constraints for the color fluxes with the fermion constraints we show that here the degrees of freedom that are relevant for long distance physics are strong coupling baryon loops, and the chemical potential couples to 3-times their temporal winding number. The interplay of the signs for the quark loops with the signs from the $\mathrm{SU}(3)$ parametrization conspires to give the baryon loops the same signs as for a single staggered fermion. The form we obtain exactly reproduces the strong coupling representation by Karsch and Mütter [11]. We conjecture that our representation of strong coupling QCD admits a fermion bag simulation, and we are currently exploring this idea.

Finally, in full QCD the color fluxes on the links receive contributions from the fermion loops and dimers, as well as the Abelian color cycles that represent the gauge degrees of freedom. Consequently also the constraints and the gauge weights couple to the combination of these two types of dual degrees of freedom. In full QCD, as well as in pure $\mathrm{SU}(3)$ gauge theory, the dual representation has the structure of a strong coupling expansion and the Abelian color flux approach allows one to calculate all coefficients of the expansion in closed form.

We conclude with stressing again that the focus of this work is on analyzing the structure of the constraints in the worldline/worldsheet representation, since this provides the manifestation of the original SU(3) gauge symmetry in the dual language. The Abelian color flux approach, combined with the binomial decomposition of the matrix elements that are sums, is a strategy that can be generalized to arbitrary non-Abelian gauge groups and several flavors of fermions. The dual worldline/worldsheet form of such systems of fermions coupled to non-Abelian gauge fields highlights different properties than the conventional representation in terms of fields, such as the manifestation of the net-particle number as the temporal winding number of the matter loops. The dual representation provides new strategies for further understanding the dynamics of non-Abelian theories and, e.g., the question how topological properties of the non-Abelian gauge fields manifest themselves in a worldsheet representation is a problem that will be addressed in the formulation presented here.

\section{ACKNOWLEDGMENTS}

We thank Falk Bruckmann, Philippe de Forcrand, Daniel Göschl, and Tin Sulejmanpasic for discussions. We acknowledge the support and the hospitality of the MITP in Mainz during the workshop DIMOCA, where part of this work was prepared. Our research is supported by the Grant No. FWF DK W1203 "Hadrons in Vacuum, Nuclei and Stars", and partly also by the FWF Grant No. I 2886N27, as well as the Grant No. DFG TR55, "Hadron Properties from Lattice QCD".
[1] S. Chandrasekharan, Proc. Sci., LATTICE2008 (2008) 003 [arXiv:0810.2419].

[2] P. de Forcrand, Proc. Sci., LAT2009 (2009) 010 [arXiv: 1005.0539].

[3] U. Wolff, Proc. Sci., LATTICE2010 (2010) 020 [arXiv: 1009.0657].

[4] C. Gattringer, Proc. Sci., LATTICE2013 (2014) 002 [arXiv: 1401.7788].

[5] R. Savit, Rev. Mod. Phys. 52, 453 (1980).

[6] H. A. Kramers and G. H. Wannier, Phys. Rev. 60, 263 (1941).
[7] K. G. Wilson, Phys. Rev. D 10, 2445 (1974).

[8] J.-M. Drouffe and J.-B. Zuber, Phys. Rep. 102, 1 (1983).

[9] P. Rossi and U. Wolff, Nucl. Phys. B248, 105 (1984).

[10] E. Dagotto, A. Moreo, and U. Wolff, Phys. Lett. B 186, 395 (1987).

[11] F. Karsch and K. H. Mütter, Nucl. Phys. B313, 541 (1989).

[12] S. Chandrasekharan and F.-J. Jiang, Proc. Sci., LAT2005 (2006) 198 [arXiv:hep-lat/0509117].

[13] P. de Forcrand and M. Fromm, Phys. Rev. Lett. 104, 112005 (2010).

[14] W. Unger and P. de Forcrand, J. Phys. G 38, 124190 (2011). 
[15] P. de Forcrand, J. Langelage, O. Philipsen, and W. Unger, Phys. Rev. Lett. 113, 152002 (2014).

[16] H. Vairinhos and P. de Forcrand, J. High Energy Phys. 12 (2014) 038.

[17] R. Leme, O. Oliveira, and G. Krein, arXiv:1703.07335.

[18] P. de Forcrand, W. Unger, and H. Vairinhos, arXiv:1710.00611.

[19] F. Bruckmann and J. Wellnhofer, Phys. Rev. D 97, 014501 (2018).

[20] O. Borisenko, V. Chelnokov, and S. Voloshyn, arXiv: 1712.03064.

[21] C. Gattringer and C. Marchis, Nucl. Phys. B916, 627 (2017).

[22] C. Marchis and C. Gattringer, Proc. Sci., LATTICE2016 (2016) 034 [arXiv:1611.01022].
[23] C. Gattringer, D. Göschl, and C. Marchis, Phys. Lett. B 778, 435 (2018).

[24] Y. Delgado Mercado, C. Gattringer, and A. Schmidt, Phys. Rev. Lett. 111, 141601 (2013).

[25] Y. Delgado Mercado, C. Gattringer, and A. Schmidt, Comput. Phys. Commun. 184, 1535 (2013).

[26] J. B. Bronzan, Phys. Rev. D 38, 1994 (1988).

[27] O. Orasch and C. Gattringer, Int. J. Mod. Phys. A 33, 1850010 (2018).

[28] M. Giuliani, O. Orasch, and C. Gattringer, arXiv: 1711.02311.

[29] S. Chandrasekharan, Phys. Rev. D 82, 025007 (2010).

[30] S. Chandrasekharan and A. Li, Phys. Rev. D 85, 091502 (2012).

[31] S. Chandrasekharan, Eur. Phys. J. A 49, 90 (2013). 